\title{
Impact of $\alpha$-synuclein fibrillar strains and B-amyloid assemblies on mouse cortical neurons endo-lysosomal logistics
}

Short title: Pathogenic assemblies and intraneuronal membrane compartments logistics

Qiao-Ling Chou ${ }^{1}$, Ania Alik², François Marquier ${ }^{1}$, Ronald Melki², François Treussart ${ }^{1}{ }^{*}$ \& Michel Simonneau ${ }^{1,3, *}$

${ }^{1}$ Université Paris-Saclay, ENS Paris-Saclay, CNRS, CentraleSupélec, LuMIn, 91190 Gif-surYvette, France.

${ }^{2}$ Laboratory of Neurodegenerative Diseases, Institut François Jacob (MIRCen), CNRS, CEA, Université Paris-Saclay, 92265 Fontenay-aux-Roses cedex, France.

${ }^{3}$ Département d'Enseignement et de Recherche en Biologie, Ecole Normale Supérieure Paris-Saclay, 91190 Gif-sur-Yvette, France.

${ }^{*}$ co-senior authors 


\section{Abstract}

Endosomal transport and positioning cooperate in the establishment of neuronal compartment architecture, dynamics and function, contributing to neuronal intracellular logistics. Furthermore, endo-lysosomal dysfunction has been identified as a common mechanism in neurodegenerative diseases. Here, we analyzed endo-lysosomal transport when $\alpha$-synuclein ( $\alpha$-syn) fibrillar polymorphs, $\beta$-amyloid (Aß) fibrils and oligomers were externally applied on primary cultures of mouse cortical neurons.

To measure this transport, we used a simple readout based on the spontaneous endocytosis in cultured neurons of fluorescent nanodiamonds, a perfectly stable nanoemitter, and the subsequent automatic extraction and quantification of their directed motions at high-throughput. $\alpha$-syn fibrillar polymorphs, $A B$ fibrils and oligomers induce a two-fold decrease of the fraction of nanodiamonds transported along microtubules, while only slightly reducing their interaction with cortical neurons. This important decrease in moving endosomes is expected to have a huge impact on neuronal homeostasis. We next assessed lysosomes dynamics, using Lysotracker. Neurons exposure to $A B$ oligomers led to an increase in the number of lysosomes, a decrease in the fraction of moving lysosome and an increase in their size, reminiscent of that found in APP transgenic model of Alzheimer's disease. We then analyzed the effect of $\alpha$-syn fibrillar polymorphs, $A B$ fibrils and oligomers on endosomal and lysosomal transport and quantified directed transport of those assemblies within cortical neurons. We report different impacts on endosomal and lysosomal transport parameters and differences in the trajectory lengths of cargoes loaded with pathogenic protein assemblies. Our results suggest that intraneuronal pathogenic protein aggregates internalization and transport may represent a target for novel neuroprotective therapeutic strategies. 


\section{Introduction}

Impairment of axonal transport has recently emerged as a factor shared by several neurodegenerative disorders (Millecamps and Julien, 2013; Morfini et al., 2009). Early impact on intraneuronal transport has been thus proposed as a phenotypic trait common to neurodegenerative diseases such as Alzheimer's, Huntington's and Parkinson's Disease (Stokin et al., 2005; Saudou and Humbert, 2016; Volpicelli-Daley et al., 2014). There is compelling evidence that abnormal protein accumulation in the brain is a key pathophysiological mechanism underlying the neurotoxicity observed in these age-related disorders (Golde et al., 203; Soto et al., 2018). Selective aggregation of misfolded proteins is a hallmark of these neurodegenerative diseases (SaezAtienzar \& Masliah, 2020). An important level of complexity is due to the fact that different species of the same molecules, such as oligomers and fibrils, contribute to a whole spectrum of toxicities (Alam et al., 2017).

Few studies have compared, within the same neurons, fibrillary and oligomeric $\alpha$ synuclein ( $\alpha$-syn) and $B$-amyloid (Aß) which are known to be involved in Parkinson and Alzheimer's diseases respectively. Brahic et al., 2016, demonstrated for instance that $\alpha$-syn, Aß42 and HTTExon1 fibrils are transported anterogradely and retrogradely in mice primary neurons grown in microfluidic chambers with different efficiencies in axons. Here, we thoroughly quantified the impact of two $\alpha$-syn fibrillar polymorphs namely fibrils $(\alpha-$ synF) and ribbons ( $\alpha$-synR), AB42 fibrils (AßF) and oligomers (AßO) on endosomal and lysosomal transports in primary cultures of mouse neurons. To measure this transport and investigate finely its parameters, we relied on our previously established method (Haziza et al., 2017), in which we let perfectly stable and non-toxic fluorescent nanodiamonds (FND) being spontaneously internalized by neurons in endosomes, then follow their displacement by fast video-microscopy and finally apply to the videos our analysis pipeline to extract and analyze single particle trajectories automatically. Using fluorescently-labelled $\alpha$-syn and $A B$ assemblies, we conducted the same investigations on their own intraneuronal transport.

Our data allow to address three complementary questions: (i) do $\alpha$-synF, $\alpha$-synR, ABF and $A B O$ influence the fraction of cargoes moving along the microtubules; (ii) do they impact the dynamics of intracellular endosomal and lysosomal transport; (iii) do they display intraneuronal transport that could illustrate pathogenic proteins assemblies spread from one neuron to another? 
We show here that all pathogenic proteins assemblies reduce the fraction of endosomes moving along microtubules and impact some of their transport parameters. Furthermore, lysosomes properties (number, fraction of lysosomes moving and transport parameters) are also affected by $A B O$. Finally, our data indicate that cargoes loaded with $\alpha$-synF, $\alpha$-synR, ABF or ABO are transported differently from endosomes, considered as control cargoes, which suggests distinct molecular characteristics of cargo-motor assemblies.

\section{Results}

\section{Quantification of intraneuronal transport using fluorescent nanodiamonds}

We quantified the intraneuronal transport with our FND tracking assay (Haziza et al., 2017). We first used a simple readout consisting in counting the number of FND detected in field-of-views (FoV) of size $40 \times 80 \mu$ m during 2 minutes. We selected each FoV based on the criteria that it contains approximately the same density of neuronal branches, as estimated from differential interference contrast images (Fig. 1). Our incubation protocol was designed to strongly limit any non-specific interactions of FND, like their attachment to the coverslip supporting the culture. We previously studied the colocalization of FND with a specific marker of the different endosomal-lysosomal compartments (Fig. 1A) 24 hours after internalization (Haziza et al., 2017). We observed that FND colocalized (fraction indicated) with endosome and lysosome at different stages of their development, each characterized by specific membrane protein markers (Haziza et al., 2017): EEA1(5\%) and Rab5 (9\%) for early endosome (EE); Rab7 (4.5\%) for late endosome; Rab11 (4.5\%) for recycling endosome (RE); LysoTracker (5\%) for the lysosome and TGN38 (3\%) for the trans Golgi network to which EE can partly fuse. The perfectly stable fluorescence of FND allows to reconstruct their trajectories accurately and identify "go" and "stop" (none or very slow motion) phases (Fig. 1B) in the transport of FND-labelled endosomes along neuronal branches as observed in differential interference contrast microscopy (Fig. 1C). 


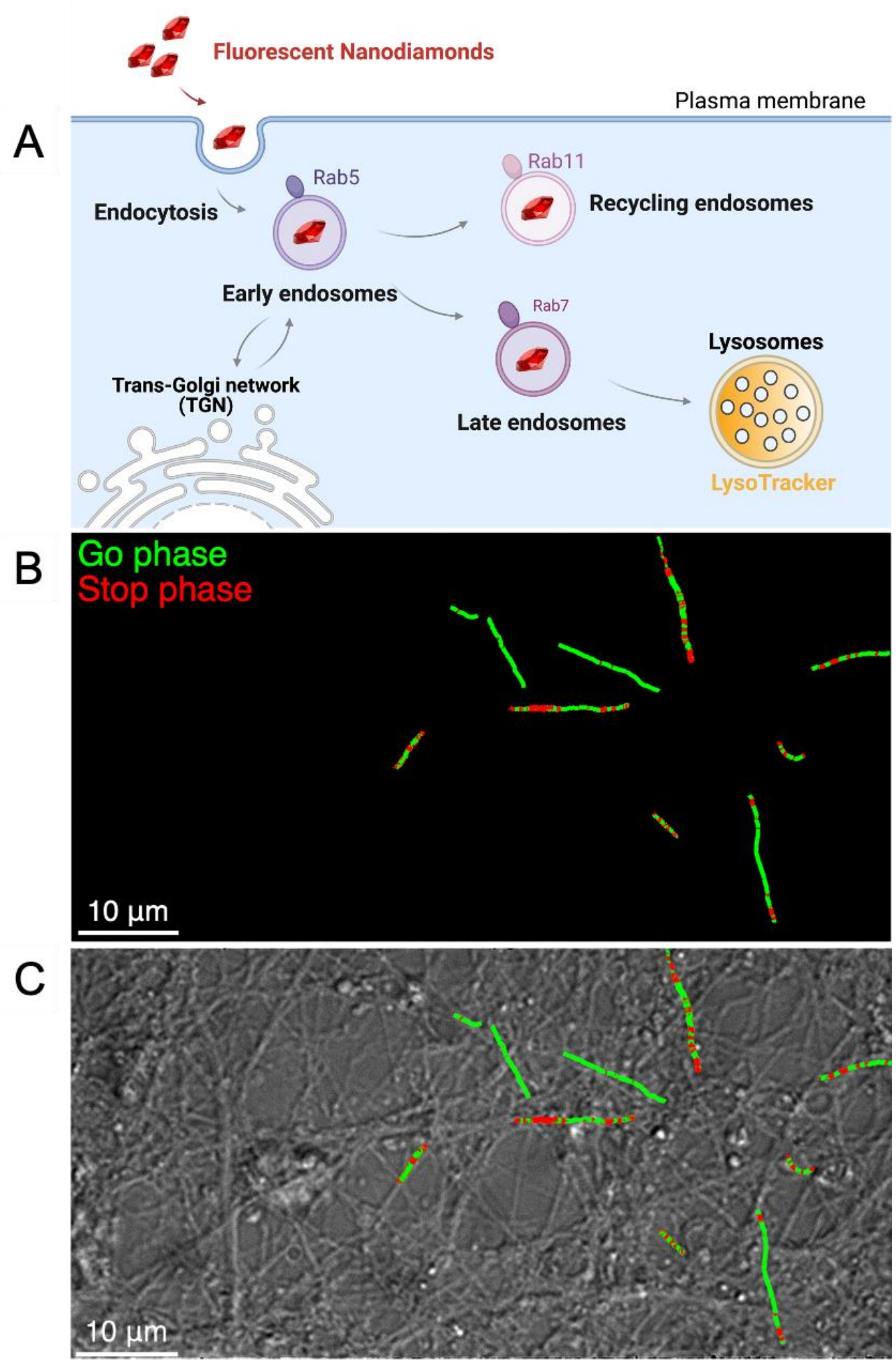

Figure 1. Recording fluorescent nanodiamond trajectories in mouse cortical neurons at DIC21. A) Schematic representation of the cargoes that are tracked thanks to FND, at different stages after their endocytosis. We previously showed (Haziza et al., 2017) that FND are present in cargos at different stage of their lifetime after endocytosis, as shown by colocalization measurements with specific membrane protein markers: Rab5 for early endosome; Rab7 for late endosome; Rab11 for recycling endosome; Lysotracker for the lysosome. B) Illustration of FND trajectories with go (green) and stop (red) phases. C) Differential interference contrast images of cortical neurons overlapped with 10 representative trajectories. Scale bar: $10 \mu \mathrm{m}$. 


\section{$\alpha$-synF and $R$ affect the number of cargos transported along microtubules without major changes in trajectory length}

Primary cultures of mouse cortical neurons were incubated at day in culture (DIC) 20 with $\alpha$-synF or $\mathrm{R}$ at a concentration of $0.2 \mu \mathrm{M}$ for 24 hours. The intraneuronal transport in these cultures was investigated at DIC21.

Both exposures to $\alpha$-synF or R led to a small decrease $(26 \%$ for $\alpha$-synF and $13 \%$ for $\alpha$-synR) in the number of FND (moving or not) present in each FoV, indicating that both fibrillar polymorphs impact the FND binding to neuronal membrane and their transport dynamics within neurons (Fig. 2A). Indeed, if we consider the fraction of these FND having a directed motion, corresponding to those being first internalized in endosomes and then taken in charge by molecular motors, we observed that it decreases by $49 \%$, upon exposure of neurons to $\alpha$-synF and $45 \%$ in the case of $\alpha$-synR (Fig. 2B). Such a large decrease in the number of cargoes transported along microtubules is expected to impair the functions of cortical neurons.

Using our established FND-based intraneuronal transport assay (Haziza et al., 2017) we detected and quantitatively analyzed the alternation of movement and pause phases of intraneuronal cargoes motion. We first measured the length of trajectories (see Material \& Methods) for control FND, FND in the presence of either $\alpha$-synF or $\alpha$ synR and we did not evidence any major changes (Fig. 2C, Fig. 2D; decrease of $4 \%$ for $\alpha$-synF and 5\% for $\alpha$-synR).

We then measured four parameters: the curvilinear velocity of each moving phase, its run length, the duration of the pauses and the pausing frequency. The velocity (Fig. 2E) and run length (Fig. 2F) increases (velocity: $31 \%$ and $38 \%$; run length: $80 \%$ and $100 \%$, for $\alpha$-synF and $\mathrm{R}$ respectively), the pausing time decreases (Fig. 2G; 40\% decrease for both fibrillar assemblies) while the pausing frequency increases (Fig. $2 \mathrm{H} ; 19 \%$ and $25 \%$, for $\alpha$-synF and $\mathrm{R}$ respectively). 
A

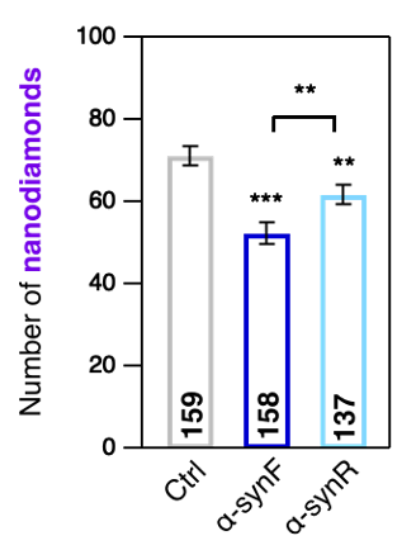

D

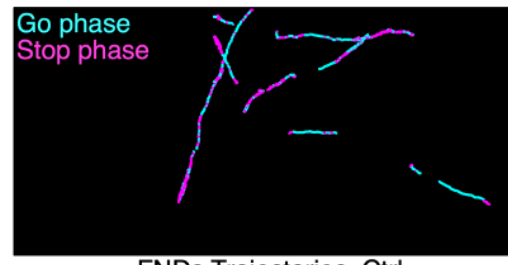

FNDs Trajectories_Ctrl
B

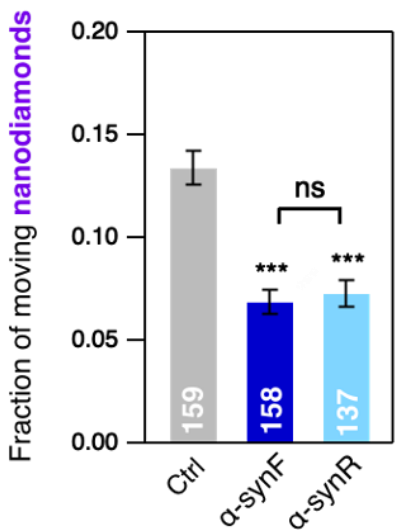

C

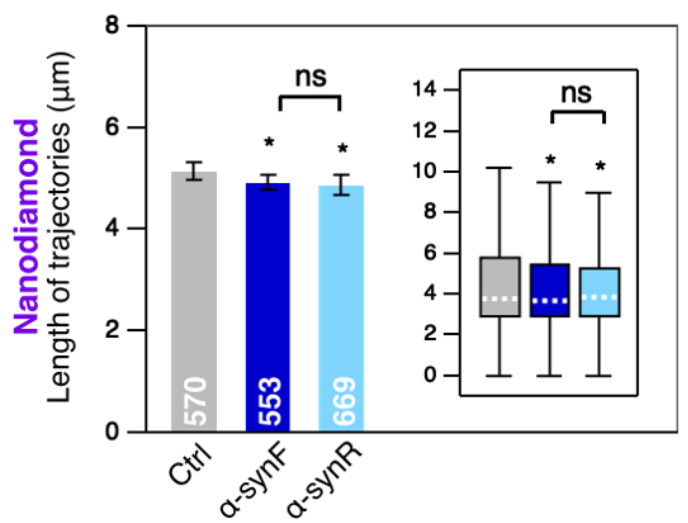

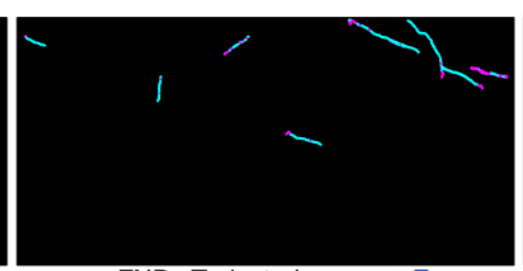

FNDs Trajectories_a-synF

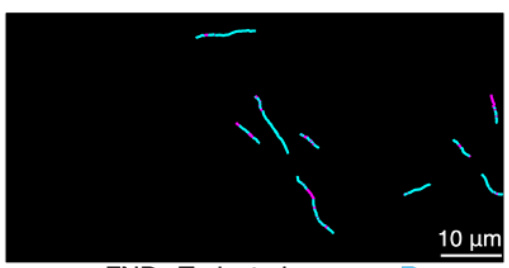

FNDs Trajectories_a-synR
E
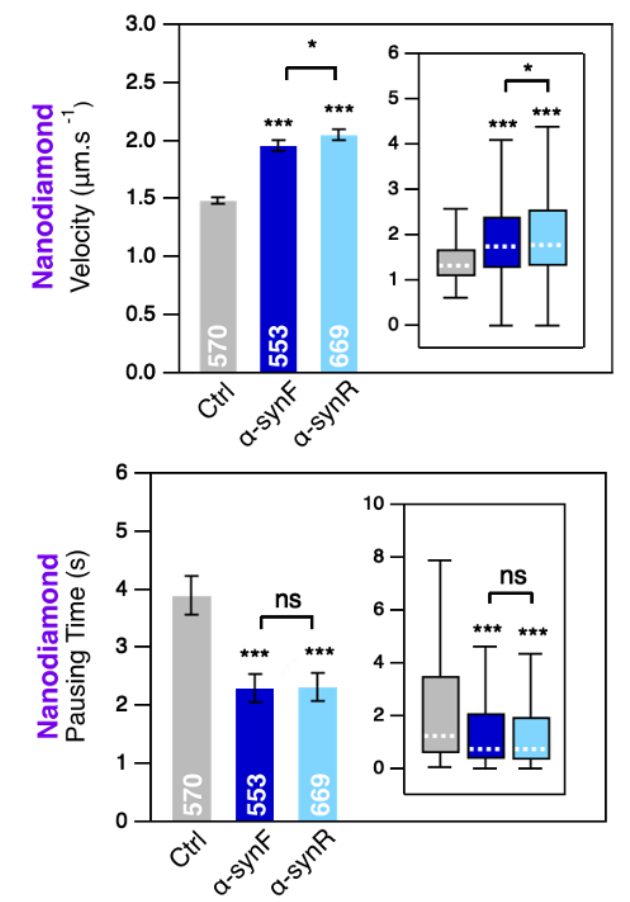

\section{G}

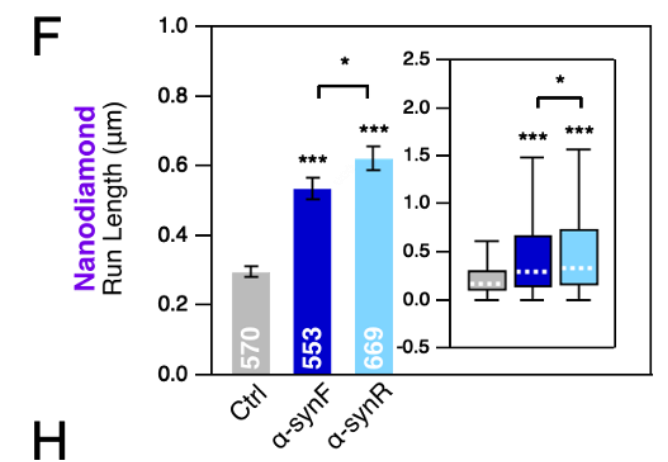

$\mathrm{H}$

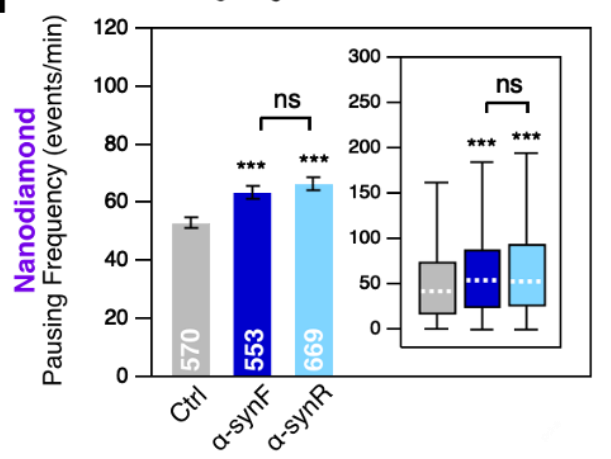

Figure 2. Effect of $\alpha$-synF or $R$ on the mobility of endosomes and their transport as measured by tracking FND-containing cargoes in mouse cortical neurons at DIC21. $24 \mathrm{~h}$ exposure to $\alpha$-synF or $\mathrm{R}$ at $0.2 \mu \mathrm{M}$ concentration, compared to nothing added control (Ctrl). A) Number of FNDs detected per field-of-view of $40 \times 80 \mu \mathrm{m}$ size during 2 mins of observation. B) Fraction of FNDs-containing cargoes having a directed motion. C) Length of FND trajectories. D) Examples of FND trajectories. Scale bar: $10 \mu \mathrm{m}$. E-H) Comparison of four transport parameters: E) curvilinear velocity, F) run length, G) pausing time and $\mathbf{H}$ ) pausing frequency. The number within each bar represents the total number of FoV $(A, B)$ or trajectories $(\mathrm{C}, \mathrm{E}-\mathrm{H})$ analyzed from $n=8$ coverslips (four independent cultures). Inset: box-plots representation of the same dataset. 
We next analyzed the same parameters for lysosomes labelled with Lysotracker red, an established marker of lysosomes, with the difference, compared to FND, that all the fluorescent spots, including the static ones, correspond to lysosomes because Lysotracker only become fluorescent once inside lysosomes. $\alpha$-synR treatment induces a slight but significant decrease (15\%) in the number of lysosomes per neurons, while $\alpha$-synF does not (Fig. 3A). This result suggests that $\alpha$-synR reduce the endocytosis. It is consistent with the observed decrease of FND interacting with neuron (Fig. 2A) that possibly reveals their reduced uptake. Furthermore, like for endosomes (Fig. 2B), a-synF and $\mathrm{R}$ induce a $46 \%$ and $32 \%$ decrease respectively (resp.) in the fraction of lysosomes having a directed motion (Fig. 3B). Analysis of lysosome trajectory lengths indicates a slight decrease (Fig. 3C; 9\% and 3\% for a-synF and $R$ resp.). Example of FoV showing lysosome trajectories in the different conditions are shown in Fig. 3D, where the large decrease in the fraction of lysosomes having a directed motion can be clearly seen.

We quantified the same transport parameters for lysosomes than for FND, using the same experimental paradigm. In contrast to what we observed for endosomes transport, exposure of neurons to $\alpha$-synF did not lead to any changes in lysosomes transport parameters (Fig. 3E-H). Interestingly however, in cortical neurons exposed to $\alpha$-synR, we measured a slight increase of $6 \%$ in lysosomes velocity (Fig. 3E) and larger one of $26 \%$ in run length (Fig. 3F), no significant change in pausing time (Fig. $3 \mathrm{G}$ ) and a slight increase of $4 \%$ in pausing frequency (Fig. $3 \mathrm{H}$ ). 
A

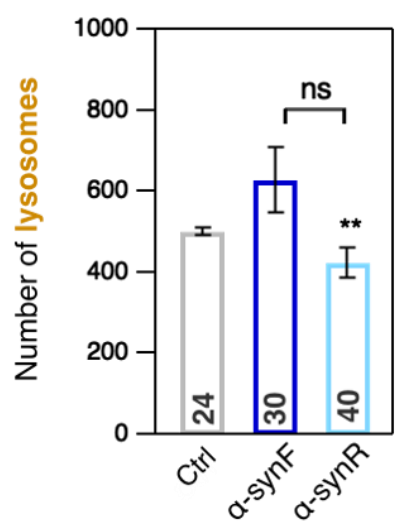

D

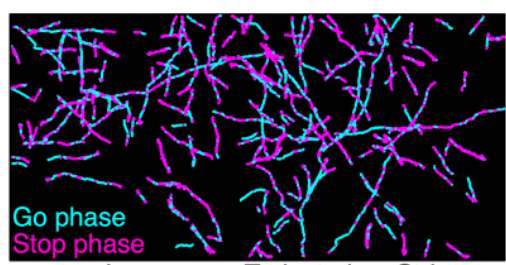

Lysosomes Trajectories_Ctrl

$E$
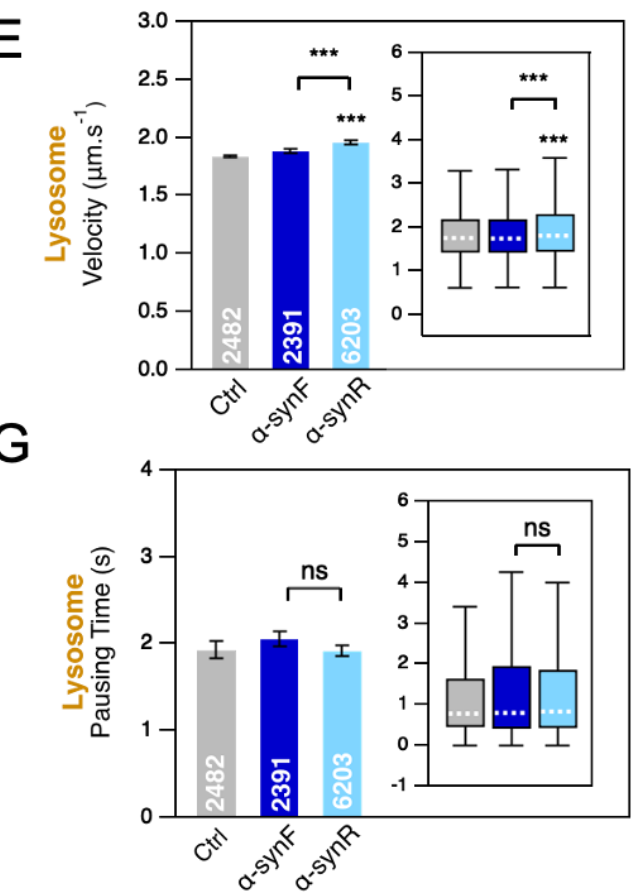

B

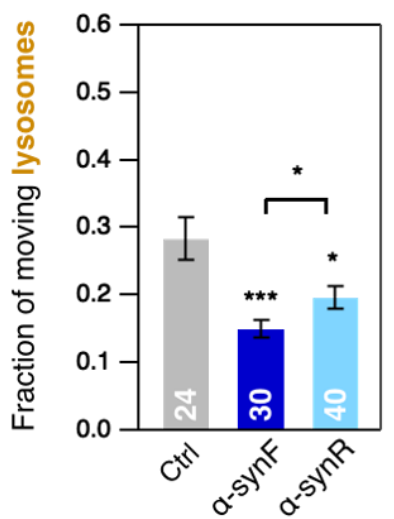

C

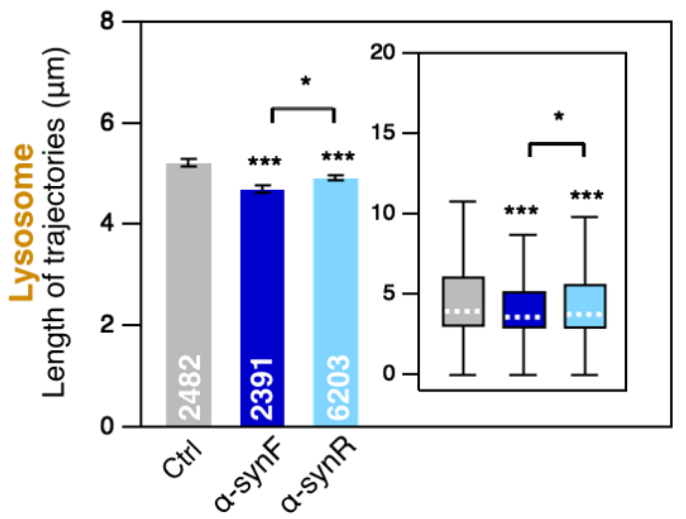

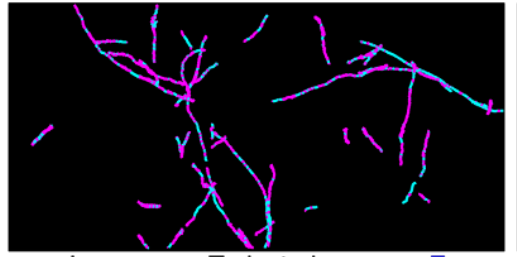

Lysosomes Trajectories_a-synF

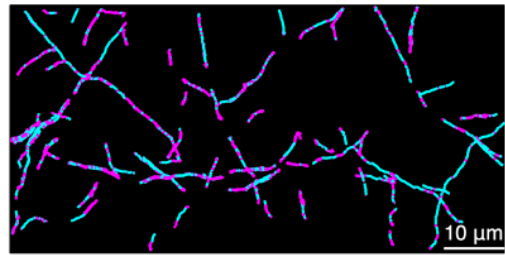

Lysosomes Trajectories_al-synt

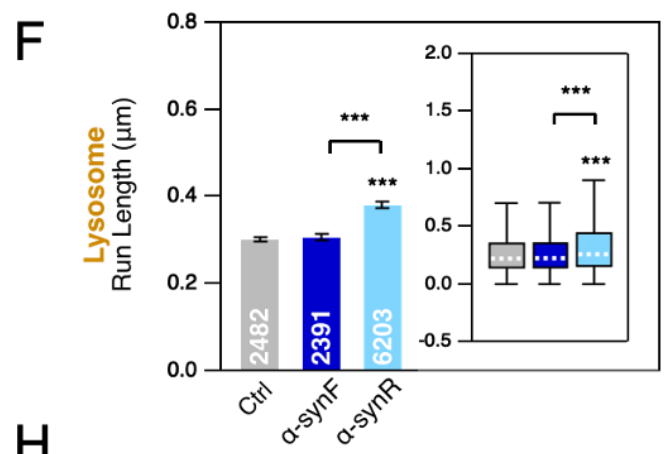

$\mathrm{H}$

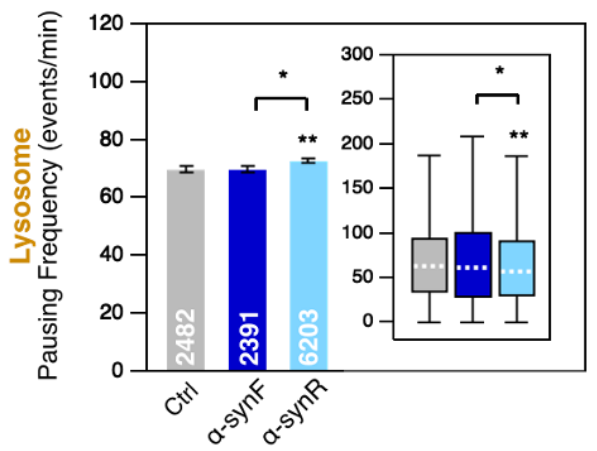

Figure 3. Effect of $\alpha$-synF or $\alpha$-synR on the mobility of lysosomes and their transport in mouse cortical neurons at DIC21. $24 \mathrm{~h}$ exposure to $\alpha$-synF or $\alpha$-synR at $0.2 \mu \mathrm{M}$ concentration, compared to nothing added control (Ctrl). A) Number of lysosomes detected per field-of-view of $40 \times 80 \mu \mathrm{m}$ size during 2 mins of observation. B) Fraction of lysosomes having a directed motion. C) Length of lysosome trajectories. D) Examples of lysosome trajectories. Scale bar: $10 \mu \mathrm{m}$. E-H) Comparison of four transport parameters: E) curvilinear velocity, F) run length, G) pausing time and $\mathbf{H})$ pausing frequency. The number within each bar represents the total number of FoV $(A, B)$ or trajectories $(C, E-H)$ analyzed from $n=2$ coverslips (from one culture). Inset: box-plots representation of the same dataset. 


\section{$A B$ assemblies affect the number of cargos transported along microtubules without major changes in trajectory length}

We also analyzed the same parameters after DIC20 mouse cortical neurons exposure to either $A B F$ or $A B O$, for 24 hours, followed by intracellular transport measurement at DIC21. We started with the same concentration of $0.2 \mu \mathrm{M}$ as for $\alpha$-syn assemblies. We could detect for both $A B F$ and $A B O$ small decreases (19\% and $18 \%$ for $A B F$ and $A B O$ resp.) in the number of FND per FoV (Suppl. Fig. S1A) and in the fraction of FND having a directed motion (Suppl. Fig. S1B; $11 \%$ and $18 \%$ for $A B F$ and $A B O$ respectively). Despite these impacts at such low concentration, we decided to continue the study with the larger concentration of $1 \mu \mathrm{M}$, since we were not able to detect any directed motion of the fluorescently labelled (ATTO 488) form of these AB assemblies at $0.2 \mu \mathrm{M}$, contrary to fluorescently-labelled $\alpha$-syn assemblies, while we want to compare these displacements. This value of $1 \mu \mathrm{M}$ of $A B$ concentration is also commonly used and has been reported to have a biological impact (Marshall et al., 2020). Figure $4 A$ shows the same slight decrease ( $3 \%$ and $7 \%$ for $A B F$ and $A B O$ resp.) of FND interacting with neurons exposed to $A B F$ or $A B O$ as for $0.2 \mu M$ concentration (Suppl. Fig. S1A), but this time accompanied by a much larger decrease (56\% and $29 \%$ for $A B F$ and $A B O$ resp.) of the fraction having directed motions (Fig. 4B). FND trajectory lengths stay almost the same for ABF ( $3 \%$ decrease) but are reduced by $\mathrm{ABO}$ (Fig. 4C, Fig. 4D; 13\% decrease). To summarize, as for $\alpha$-syn assemblies (Fig. 3), the exposure of cortical neurons to $A B$ assemblies induce important and significant decreases of the endosomal transport.

We then measured more precisely the impact of $A B F$ and $A B O$ on endosomal transports parameters. We observed an increase of FND velocity (Fig. 4E; 20\% and $15 \%$ for $A B F$ and $A B O$ resp.) and run length (Fig. $4 F ; 5 \%$ and $7 \%$ for $A B F$ and $A B O$ resp.), a decrease in their pausing time (Fig. 4G; $36 \%$ and $12 \%$ for $A B F$ and $A B O$ resp.) and an increase of the pausing frequency (Fig. $4 \mathrm{H} ; 28 \%$ and $15 \%$ for $A B F$ and $A B O$ resp.), with effects more pronounced for $A ß F$ than for $A ß O$. Interestingly, the same trends of changes were also observed at the lower $A B F$ and $A B O$ concentration of $0.2 \mu \mathrm{M}$ (Suppl. Fig. S2). Let us finally point out that for ABF, the important changes of some transport parameters overall combine in an only very slight decrease in trajectory length as shown in Fig 4C, which makes the detailed quantitative analysis performed all the more useful. 
A

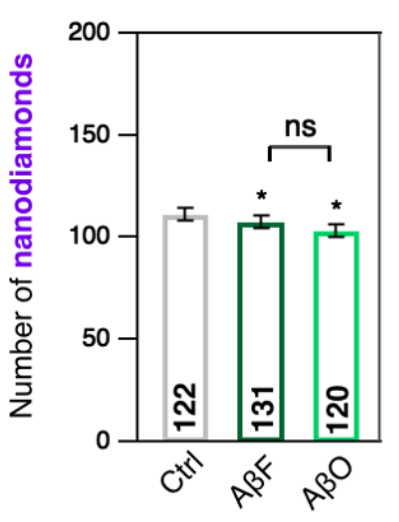

B

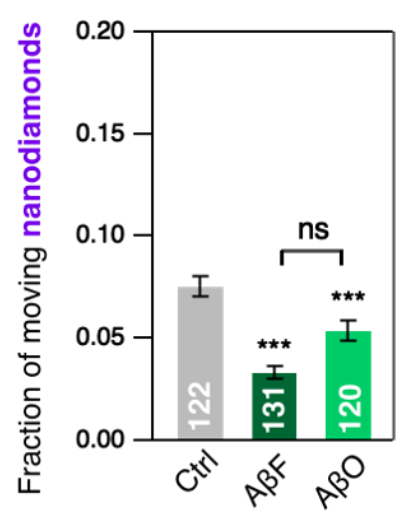

C

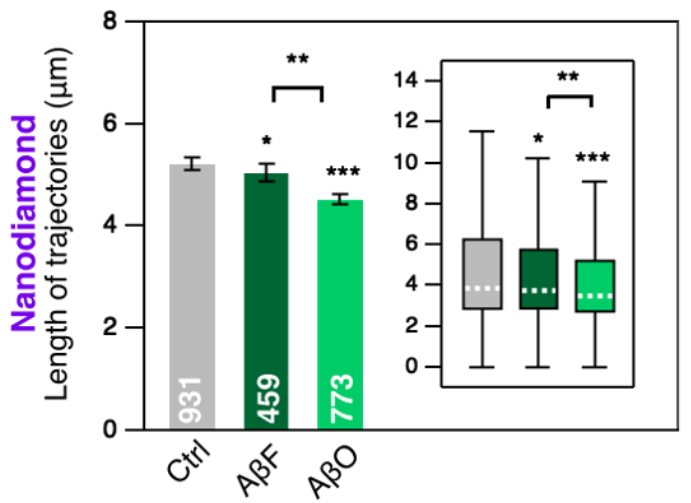

D

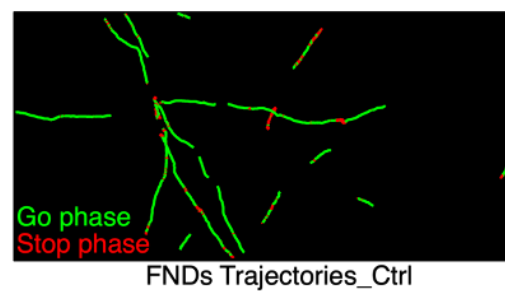

$\mathrm{E}$

G
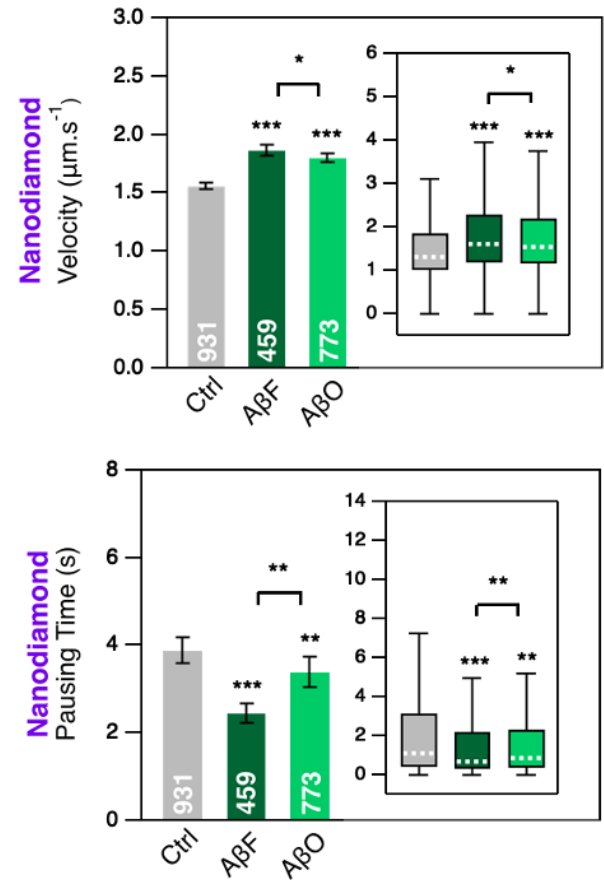

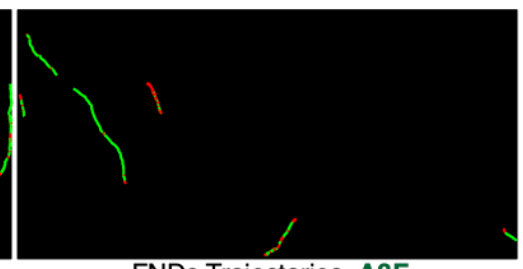

FNDs Trajectories_ABF

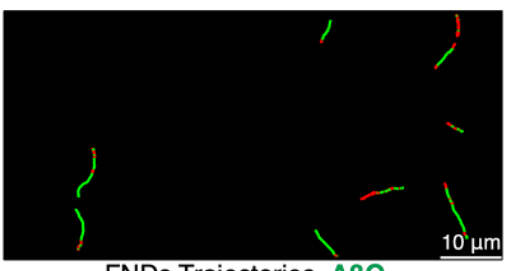

FNDs Trajectories_ABO

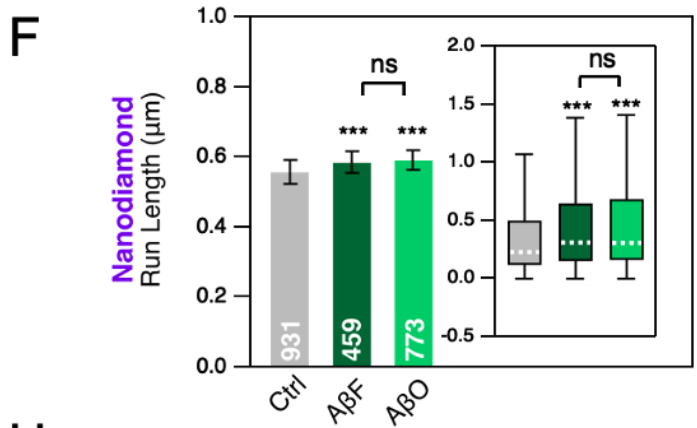

$\mathrm{H}$

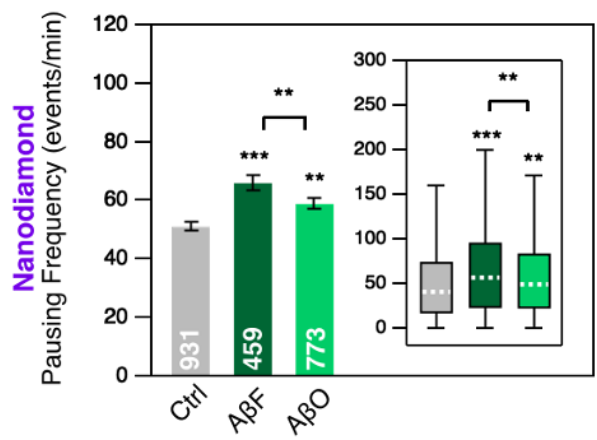

Figure 4. Effect of $A B F$ and $A B O$ on the mobility of endosomes and their transport as measured by tracking FND-containing cargoes in mouse cortical neurons at DIC21. $24 \mathrm{~h}$ exposure to ABF and $A B O$ at $1 \mu \mathrm{M}$ concentration, compared to nothing added control (Ctrl). A) Number of FNDs detected per field-of-view of $40 \times 80 \mu \mathrm{m}$ size during 2 mins of observation. B) Fraction of FNDs-containing cargoes having a directed motion. C) Length of FND trajectories. D) Examples of lysosome trajectories. Scale bar: $10 \mu \mathrm{m}$. E-H) Comparison of four transport parameters: E) curvilinear velocity, F) run length, G) pausing time and $\mathbf{H}$ ) pausing frequency. The number inside the bar represents the total number of FoV $(A, B)$ or trajectories $(C, E-H)$ analyzed from $n=6$ coverslips (three independent cultures). Inset: boxplots representation of the same dataset. 
Regarding the impact of $A B$ on lysosomal transport, we noticed large differences between the two types of assemblies $A B F$ and $A B O$. In neurons exposed to $A B O$, the total number of lysosomes detected in a FoV as compared to controls increased by $50 \%$ (Fig. 5A) while it stayed unchanged in case of exposure to AßF. The intracellular transport measurements we performed showed that the fraction of moving lysosomes decreased by 1.6-fold and 5.7-fold in neurons exposed to $A B F$ and $A B O$, respectively (Fig. 5B; from $23 \%$ for control to $14 \%$ for $A B F$ and $4.5 \%$ for $A B O$ ). Lysosome trajectory lengths were only slightly decreased for $\mathrm{ABF}(5 \%)$ and more significantly reduced for $\mathrm{A} O$ (Fig. 5C and Fig. 5D; 17\% decrease) exposure.

We also measured lysosome transport parameters in the presence of $1 \mu \mathrm{M} A B F$ or $A B O$ (Fig. 5E-H). For $A B F$, we observed almost no change in velocity (Fig. 5E), pausing time (Fig. 5G), and pausing frequency (Fig. $5 \mathrm{H}$ ). In contrast, exposure to $A B O$, led to a 1.7-fold increase of the pausing time. The run length decreased significantly for both assemblies (Fig. 5F; with 9\% and 13\% decrease for $A B F$ and $A B O$ exposure resp.).

Furthermore, as changes in lysosome size was described in APP mouse transgenic model of Alzheimer's disease (Gowrishankar et al., 2015), we asked if detectable changes in lysosome diameter can be quantified upon 24 or $48 \mathrm{~h}$ exposure to $1 \mu \mathrm{M}$ $A B F$ and $A B O$. We detected a slight increase $(7 \%)$ of lysosome diameter upon exposure of neurons to $A B F$ at $24 \mathrm{~h}$ that disappears at $48 \mathrm{~h}$. This contrasts with the increase we observed at both time points (11\% and $7 \%$ at $24 \mathrm{~h}$ and $48 \mathrm{~h}$ resp.) in neurons exposed to $1 \mu \mathrm{M} A B O$ (Fig. 5I-J).

The finding that $\mathrm{ABO}$ addition triggers an increase in lysosomes number and size and a decrease in lysosome movements is in agreement with previous reports (Gowrishankar et al., 2015; Marshall et al., 2020). 

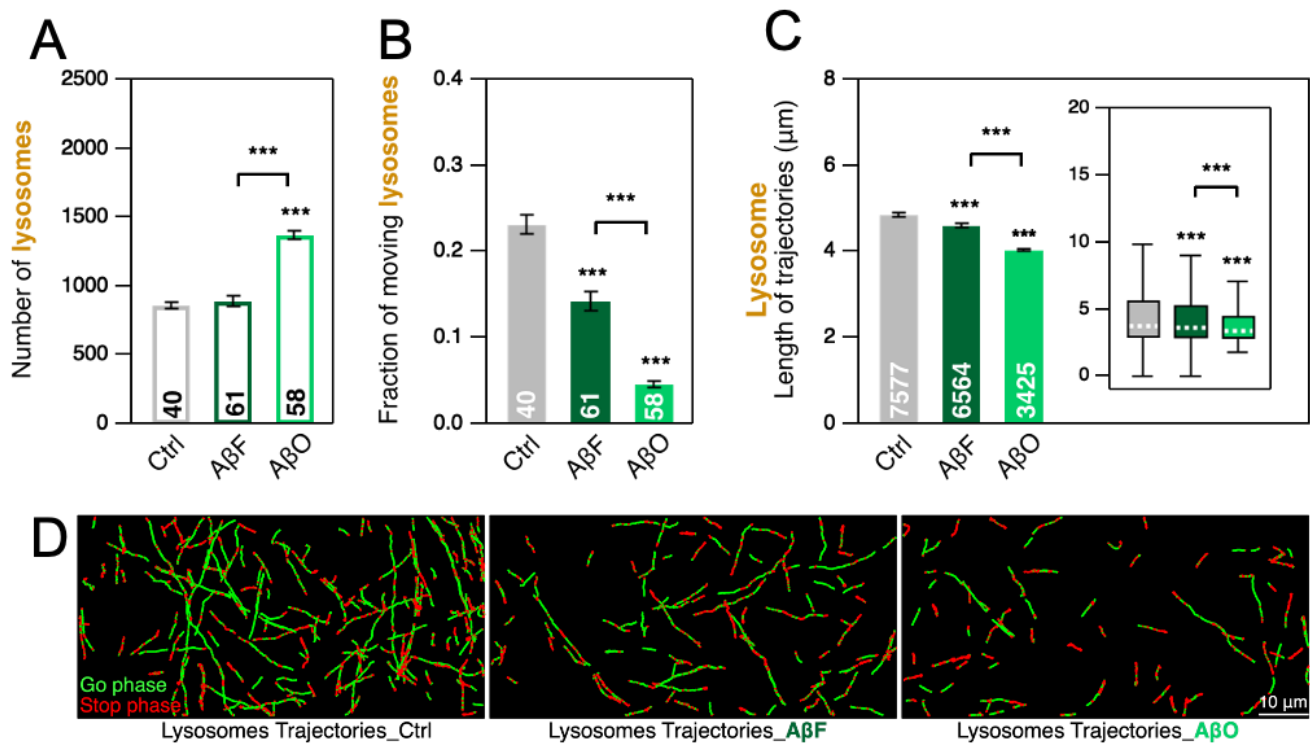

E

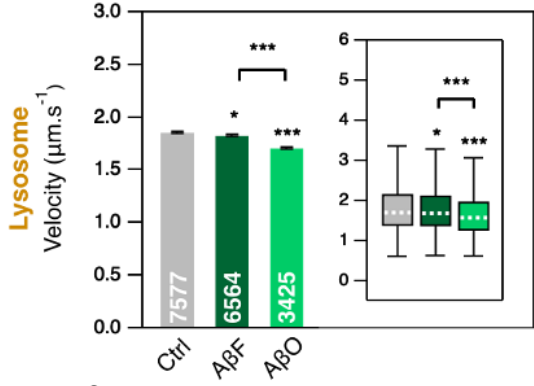

G
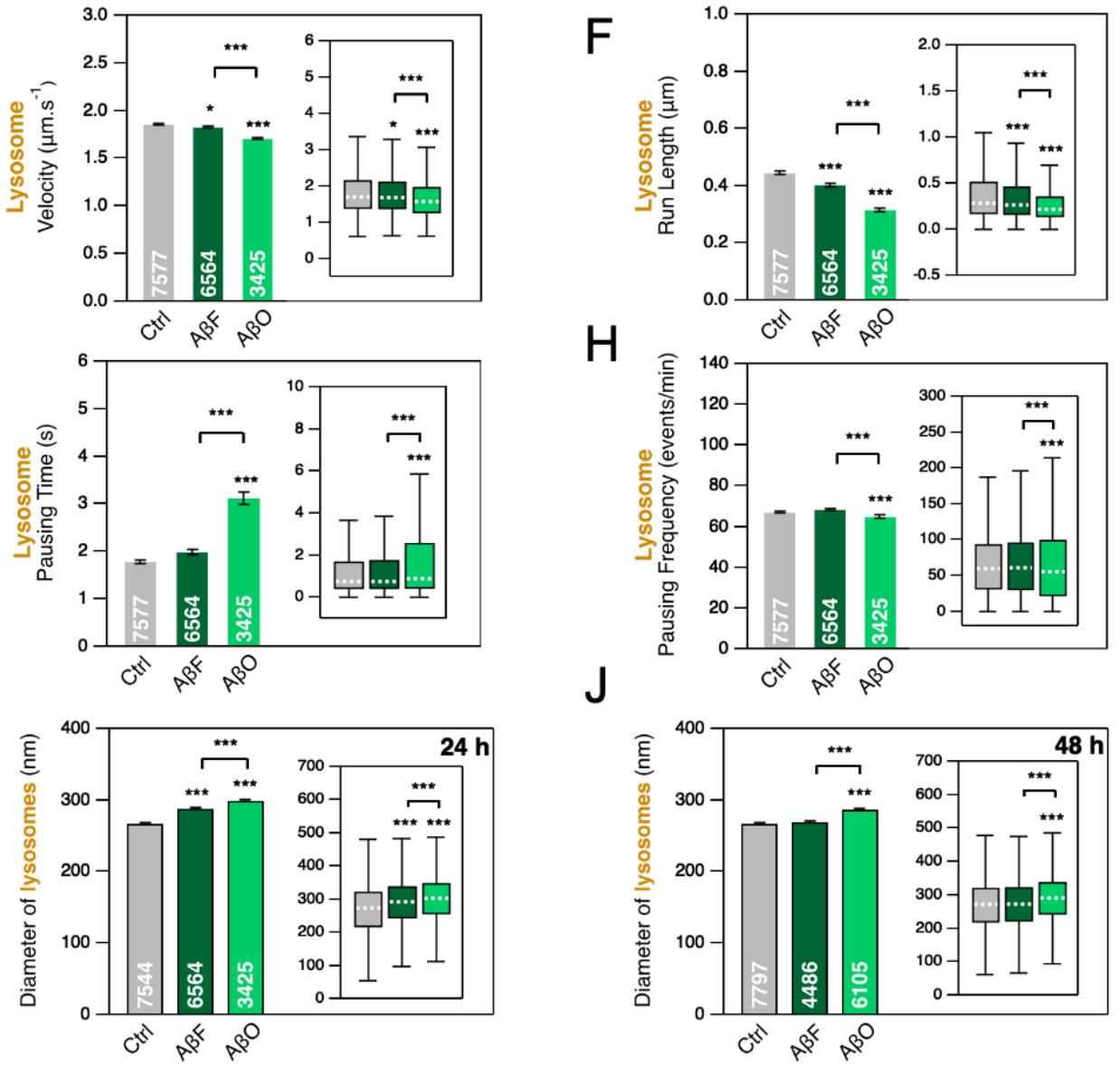

Figure 5. Effect of $A B F$ and $A B O$ on the mobility of lysosomes and their transport as measured by tracking FND-containing cargoes in mouse cortical neurons at DIC21. $24 \mathrm{~h}$ exposure to ABF and $A B O$ at $1 \mu \mathrm{M}$ concentration, compared to nothing added control. A) Number of lysosomes detected per field-of-view of $40 \times 80 \mu \mathrm{m}$ size during 2 mins of observation. B) Fraction of lysosomes having a directed motion. C) Length of lysosome trajectories. D) Examples of lysosome trajectories. Scale bar: $10 \mu \mathrm{m}$. E-H) Comparison of four transport parameters: E) curvilinear velocity, F) run length, G) pausing time and $\mathbf{H}$ ) pausing frequency. The number inside the bar represents the total number of FoV $(A, B)$, trajectories $(\mathrm{E}-\mathrm{H})$ and lysosomes $(\mathrm{I}, \mathrm{J})$ analyzed from $n=2$ coverslips (from one culture). Inset: box-plots representation of the same dataset. 


\section{Transport of $\alpha$-syn and $A \beta$ assemblies within cortical neurons.}

We also assessed $\alpha$-syn and $A B$ assemblies transport within cortical neurons while documenting their impact on endosomes and lysosomes dynamics. As ATTO 488 dye used to label $\alpha$-syn and $A B$ assemblies exhibit no emission spectrum overlap with neither FND nor Lysotracker deep red, we were able to measure simultaneously the transport properties of endosome or lysosome and the assemblies on two-color channels.

We first studied $\alpha$-syn fibrillar assemblies transport (Fig. 6). We found that $\alpha$-synF and $R$ display directed movements as shown by examples of trajectories in Fig. 6A-B. We compared these motions to the endosomal transport in the presence of $\alpha$-syn fibrillar polymorphs. Interestingly, $\alpha$-syn F and R trajectories are about $29 \%$ shorter than those of FND (Fig. 6C). We also compared the transport parameters and found smaller velocity (Fig. 6D; $5 \%$ and $10 \%$ for $\alpha$-synF and $\alpha$-synR resp.), run length (Fig. 6E; 39\% and $46 \%$ for $\alpha$-synF and $\alpha$-synR resp.) and pausing time (Fig. 6F; $19 \%$ and $21 \%$ for $\alpha$ synF and $\alpha$-synR resp.) for $\alpha$-synF and $\alpha$-synR-loaded cargoes compared to those of FND-containing endosomes. On the contrary, $\alpha$-synF and $R$ pausing frequencies were larger than those of FND (Fig. 6G; $19 \%$ and $8 \%$ for $\alpha$-synF and $\alpha$-synR resp.).

The shorter trajectories length and run-length together with the larger pausing frequency, suggest that cargoes loaded with $\alpha$-synF- and $\mathrm{R}$ are transported less efficiently than those containing FND.

Similarly, we also investigated $A B F$ and $A B O$ intraneuronal transport (Fig. 7). Both species exhibit directed transport as shown by examples of trajectories in Fig. 7A-B. These trajectories are shorter than the ones of FND in the same conditions (Fig. 7C), as for $\alpha$-syn fibrillar assemblies.

Regarding the transport parameters, compared to FND, $A B F$ and $A B O$ have slightly larger velocity (Fig. 7D; $8 \%$ and $12 \%$ for $A B F$ and $A B O$ resp.), and a trend towards a shorter run-length (Fig. 7E; $7 \%$ and $28 \%$ for $A B F$ and $A B O$ resp.). As $\alpha$-syn fibrillar assemblies, $A B F$ and $A B O$ exhibit a much shorter pausing time (Fig. $7 F ; 49 \%$ and $53 \%$ for $A B F$ and $A B O$ resp.) and a much larger pausing frequency (Fig. 7G; 41 and $36 \%$ for $A B F$ and $A B O$ resp.).

As for $\alpha$-syn fibrillar assemblies the shorter trajectories length and run-length together with the larger pausing frequency suggest that cargoes loaded with $A B F$ and $A B O$ are transported less efficiently than those containing FND. 


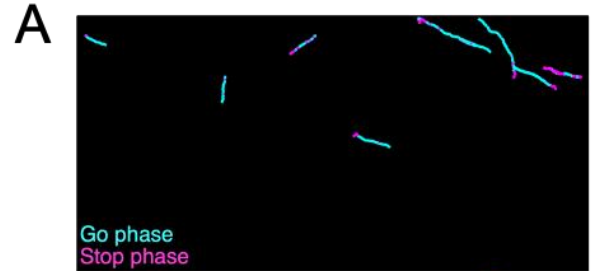

FNDs Trajectories_a-synF

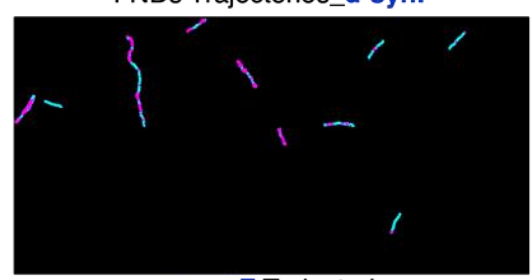

a-synF Trajectories

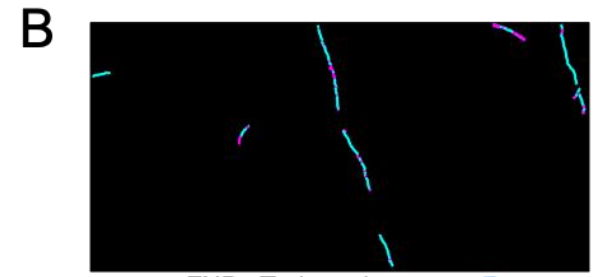

FNDs Trajectories_a-synR

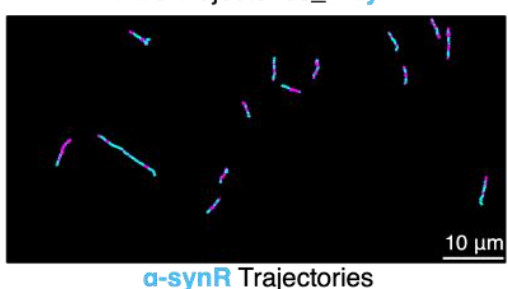

a-synR Trajectories
C

D

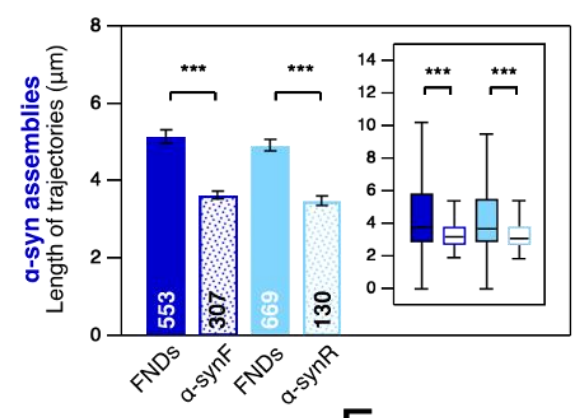

E
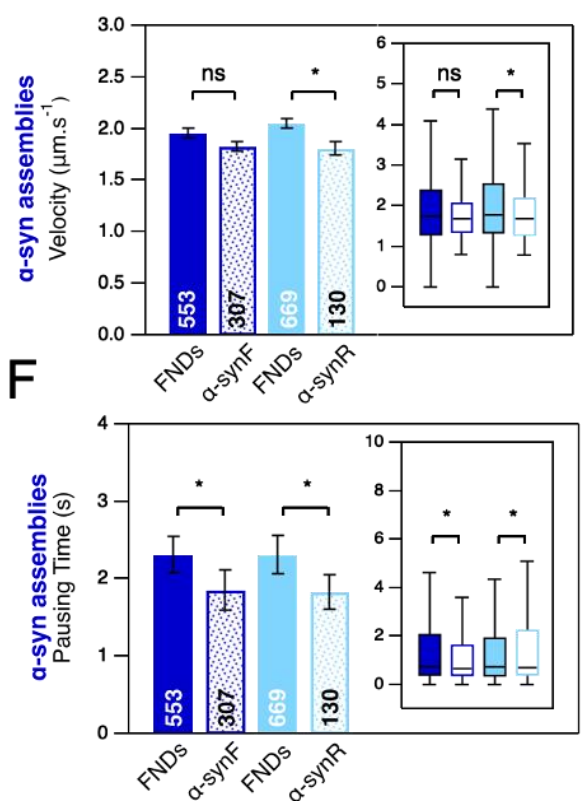
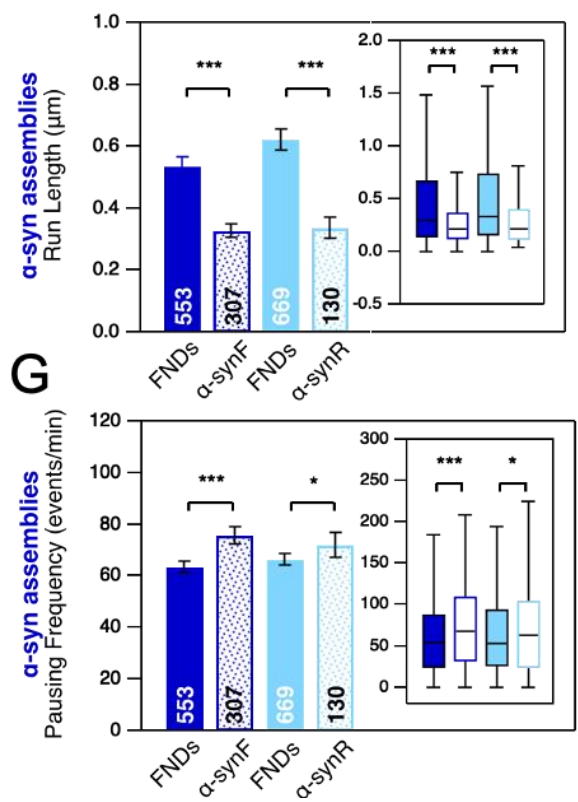

Figure 6. Intraneuronal transport of ATTO 488-labeled $\alpha$-synF and R in mouse cortical neurons at DIC21. DIC20 cortical neurons were exposed to $\alpha$-synF and R during $24 \mathrm{~h}$, at concentration of $0.2 \mu \mathrm{M}$. A-B) Examples of $\alpha$-synF and R, and FND trajectories (in the presence of $\alpha$-synF and R). Scale bar: $10 \mu \mathrm{m}$. C) Length of $\alpha$-synF, $\alpha$-synR and FND trajectories. D-G) Comparison of four transport parameters: D) curvilinear velocity, E) run length, F) pausing time and $\mathbf{G}$ ) pausing frequency. The number inside the bar represents the total number of trajectories analyzed from $n=8$ coverslips (four independent cultures) is indicated in each bar. Inset: box-plots representation of the same dataset. 


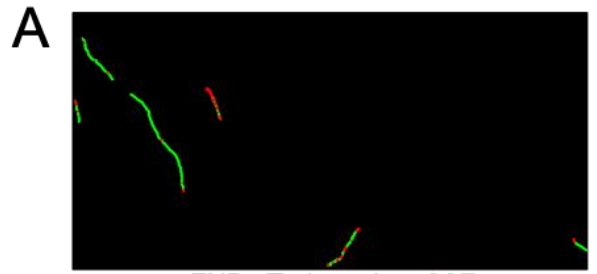

FNDs Trajectories_A $\mathrm{A} F$

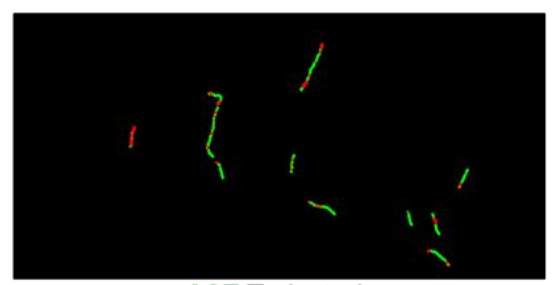

A $\mathrm{BF}$ Trajectories

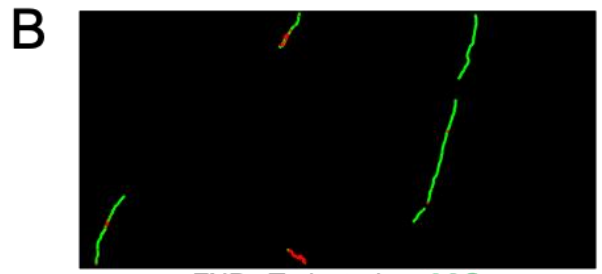

FNDs Trajectories_ABO

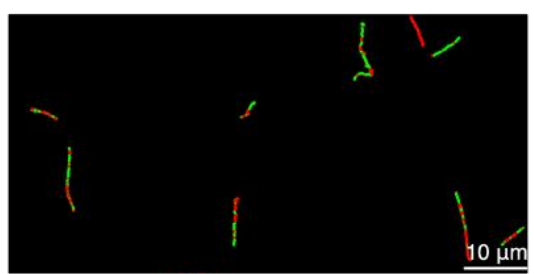

AßO Trajectories

C

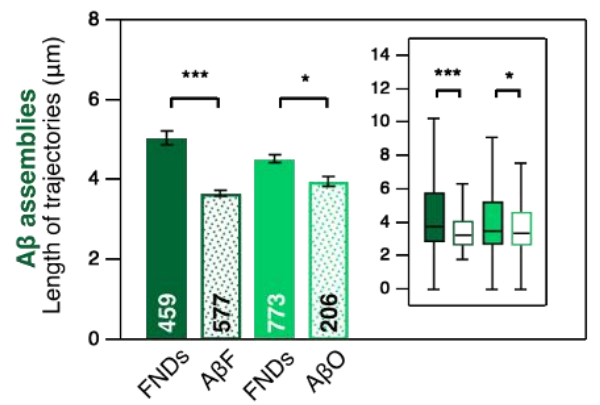

$\mathrm{D}$
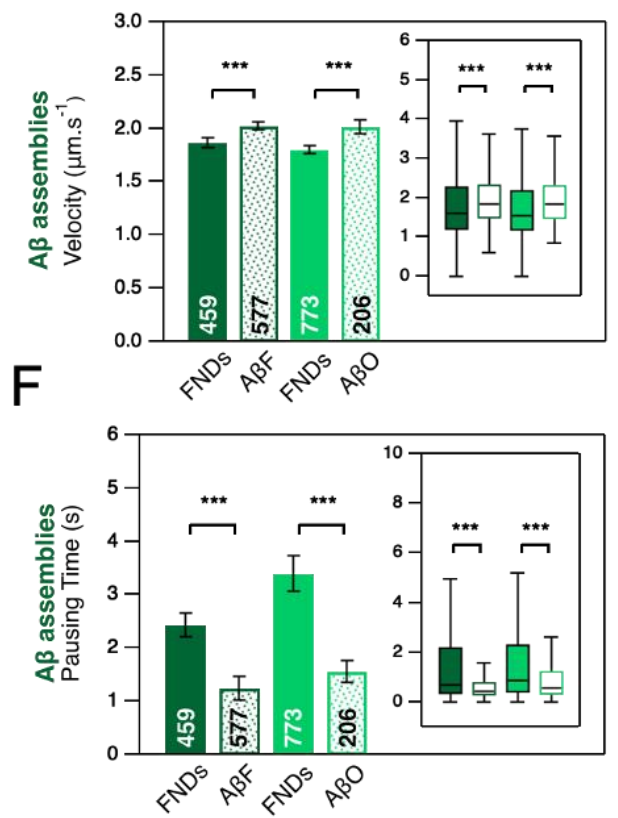

$\mathrm{E}$
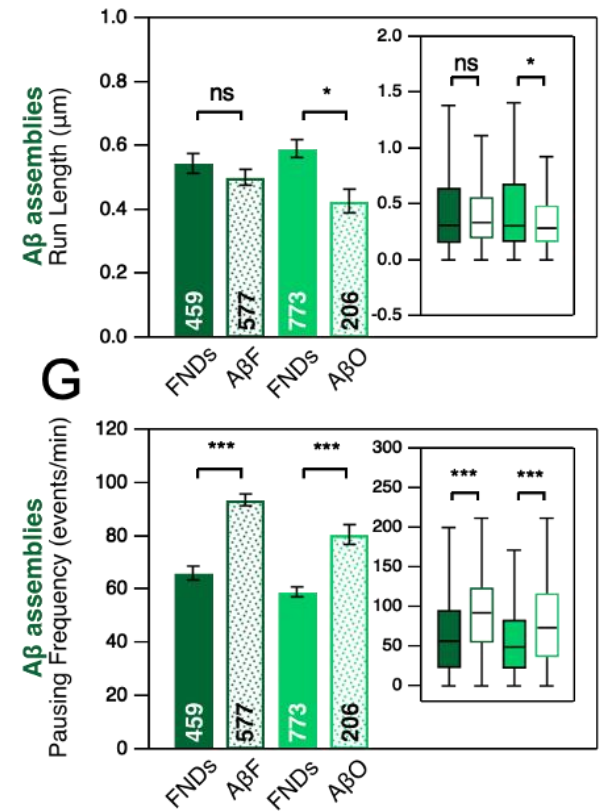

Figure 7. Intraneuronal transport of $A T T O$ 488-labeled $A B F$ and $A B O$ in mouse cortical neurons at DIC21. DIC20 cortical neurons were exposed to $A B F$ and $A B O(1 \mu \mathrm{M})$ during $24 \mathrm{~h}$. A-B) Examples of trajectories. Scale bar: $10 \mu \mathrm{m}$. C) Length of $A B F, A B O$ and FND trajectories. D-G) Comparison of four transport parameters: D) curvilinear velocity, E) run length, F) pausing time and G) pausing frequency. The number inside the bar represents the total number of trajectories analyzed from $n=6$ coverslips (from three independent cultures) is indicated in each bar. Insets: box plots representation of the same dataset. 
Finally, we assessed in a quantitative manner the co-localization of $\alpha$-syn and $A B$ assemblies with lysosomes, as a function of neuron exposure time 24,48 and 72 hours (Suppl. Fig. S3 and S4). While the fraction of $\alpha$-syn fibrillar assemblies moving within lysosomes increases from $\approx 4 \%$ at $24 \mathrm{~h}$ to $12-14 \%$ at $72 \mathrm{~h}$, it reaches already $\approx 41 \%$ at $24 \mathrm{~h}$ for $\mathrm{A} ß$ fibrils continuing its increase up to $\approx 51 \%$ at $48 \mathrm{~h}$. $\mathrm{A} O \mathrm{O}$ moving in or with lysosomes have slightly lower colocalization proportions, however much larger than for $\alpha$-syn fibrillar assemblies.

\section{DISCUSSION}

\section{Potential consequences of a decrease in the number of cargos transported at a given time in a cortical neuron.}

The exposure of cortical neurons to $\alpha$-syn and $A B$ assemblies led to important reductions (between $32 \%$ and $56 \%$ ) of moving endosomes and lysosomes along neuronal branches (Figs. 1-2). We previously showed that pathogenic $\alpha$-syn and $A B$ assemblies bind the plasma membrane with, as a consequence, a redistribution of essential membrane proteins (Renner et al. 2010; Shrivastava et al., 2013; Shrivastava et al., 2015). We further reviewed the physico-pathogenic mechanisms at the origin and resulting from pathogenic proteins assemblies-plasma membrane components interactions (Shrivastava et al 2017). The reduction we report here might be due to changes in membrane dynamics and endocytosis rate.

Exposure of cortical neurons to $\alpha$-syn and $A B$ assemblies affected moving FNDcontaining endosome properties. $\alpha$-syn fibrillar assemblies increased their velocity by $31-38 \%$ and run length by $80-100 \%$ (Fig. 2E-F) and decreased their pausing time by $40 \%$ (Fig. 2G) while increasing their pausing frequency to a lesser extend $(19-25 \%$, Fig. $2 \mathrm{H}$ ). We observed similar effects but less pronounced for moving FND-containing endosomes when neurons are exposed to $A B F$ or $A B O$ (Fig. 4E-H). These changes reflect an increase in mobility of moving FND-containing endosomes. Hence, while decreasing the fraction of moving FND-labeled endosomes, $\alpha$-synF and R or AßF and $A B O$, increase the overall mobility of the moving ones.

Furthermore, considering that lysosomes and late endosomes act as mRNA translation platforms (Cioni et al., 2019; Liao et al., 2019; Fernandopulle et al., 2021), changes in the number of cargos transported at a given time within a cortical neuron is expected to dramatically impact mRNA translation platform either in dendrites or in axons. 


\section{Effect of $\alpha$-syn and $A B$ assemblies on lysosomes transport}

We observed that $\alpha$-synF (Fig. 3E-H) and AßF (Fig. 5E-H) barely impact the lysosomal transport parameters as compared to the control. In contrast, $\alpha$-synR (Fig. 3E-H) and $\mathrm{ABO}$ (Fig. 5E-H) induce significant changes of lysosomes transport parameters. Furthermore, the size of lysosomes significantly increased in the presence of $A B O$ (Fig. 5I-J).

This set of impairments of lysosomal transport in Alzheimer-related context are in full agreement with previous reports (Gowrishankar et al., 2015; Marshall et al., 2020). Indeed, using a mouse models of Alzheimer's disease, Gowrishankar et al., 2015 evidenced axonal lysosome accumulations with local impairment in the retrograde axonal transport of lysosome precursors. Similarly, (Marshall et al., 2020) found that misfolded Aß42 impacts the endo-lysosomal pathway. They reported impairments in the uptake of proteins that use a dynamin-dependent endosomal mechanism and accumulation of lysosomes.

\section{Intraneuronal transport of neurodegenerative-related molecular species}

We were able to quantity the intraneuronal transport parameters for $\alpha$-syn and $A B$ pathogenic species (Figs 6-7). We found that cargoes loaded with $\alpha$-synF and R exhibit a more dynamical transport compared to those containing FND that are characterized by a larger pausing frequency and shorter run-length and pausing time (Fig. 6D-G).

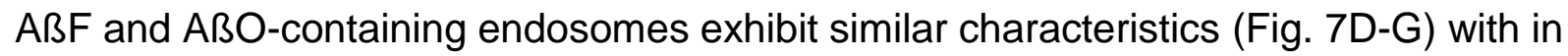
addition an increase in velocity, not observed for $\alpha$-synF and $R$. These results suggest that similar molecular mechanisms are at play in the transport of the two $\alpha$-syn fibrillar polymorphs, the $A ß F$ and $A ß O$. However, the $\approx 7$-fold larger fraction of $A B F$ and $A B O$ found in moving lysosomes compared to $\alpha$-syn assemblies (Fig. S4), also indicate differences in the molecular interactions of $A B$ assemblies with lysosomes.

These results suggest either a cellular triage leading to differential transport of $\alpha$-syn and $A B$ assemblies.

\section{Potential druggable targets}

Two main targets can be underscored from the results presented here: the decrease in the number of cargoes transported at a given time inside a cortical neuron and the intraneuronal transport of pathogenic protein assemblies.

We observed a large decrease in the fraction of transported cargoes (endosomes and lysosomes) for all pathogenic protein assemblies (Figs. 2B, 3B, 4B and 5B), in the 
order of $30-50 \%$, and up to 5-fold for lysosomes in neurons exposed to ABO (Fig. 5B). Such a large decrease is likely to impact the physiology of neurons and it is reasonable to consider that the transport of other cargoes, such as mitochondria and RNA granules, is also affected.

The cargoes transport blockade within cortical neurons we report could either result from a direct interaction between pathogenic aggregates and the intraneuronal transport machinery or a pathogenic aggregates-mediated transcriptional changes in transport proteins expression (Encalada and Goldstein, 2014, Lee et al., 2014; Guo et al., 2020).

Analysis of neuronal immuno-precipitates of $\alpha$-syn fibrillar assemblies and $A B$ polymorphs may be instrumental to identify the molecular mechanisms involved in cargoes transport. A recent postmortem proteomics study identified proteins whose abundance changed at different stages of Alzheimer's disease (Li et al., 2021). At its early stage, differentially expressed proteins of "clathrin-coated endocytic vesicle membrane" (GO: 0030669) and the secretory pathway (R-HSA-432720: "Lysosome Vesicle Biogenesis" and R-HSA-432722: "Golgi Associated Vesicle Biogenesis") classes were over-represented. Comparison of proteome profile changes in our neuronal model with (Li et al., 2021) profiles can be instrumental to identify druggable targets in order to enhance the number of transported cargoes.

Finally, we quantified intraneuronal transport of neurodegenerative-linked molecular species ( $\alpha$-syn fibrillar polymorphs, $A \beta F$ and oligomers) whose transport characteristics are distinct from those of endosomes but for which no molecular characterization is yet available. Furthermore, we observed that all these protein assemblies are transported intracellularly in cortical neurons with very similar quantitative characteristics. We could not detect differences in their transport parameters. Further work will be required to identify a possible common transport mechanism and the identification of specific molecules involved in this transport can allow to selectively inhibit it.

These results also need to be considered from the standpoint of the prion-like spread of pathogenic protein particles between neurons (Brundin et al., 2010; Hardy and Revesz, 2012; Jucker and Walker, 2018). Selective inhibition may avoid the spread of these neurotoxic species. Thus, advances in the identification of targets involved in cargoes loaded with pathogenic protein aggregates transport may lead to novel neuroprotective therapeutic avenues. 


\section{Acknowledgments}

This work was supported the Joint Program on Neurodegenerative Disease Research and Agence National de la Recherche (contract TransPathND ANR-17-JPCD-0002) to M.S. and R.M. and the Fondation pour la Recherche Médicale (contract ALZ201912009776) to R.M. Q-L.C was supported by fellowships from University ParisSaclay, Taiwan Ministry for Research and JPND TransPathND (ANR-17-JPCD-0002).

\section{REFERENCES}

Alam P, Bousset L, Melki R, Otzen DE. a-synuclein oligomers and fibrils: a spectrum of species, a spectrum of toxicities. J Neurochem. 2019 Sep;150(5):522-534.

Brahic M, Bousset L, Bieri G, Melki R, Gitler AD. Axonal transport and secretion of fibrillar forms of $\alpha$-synuclein, A 42 peptide and HTTExon 1. Acta Neuropathol. 2016 Apr;131(4):539-48.

Braak H, Braak E: Neuropathological stageing of Alzheimer-related changes. Acta Neuropathol 1991, 82:239-259.

Braak H, Del Tredici K, Ru'b U, de Vos RAI, Jansen Steur ENH,Braak E: Staging of brain pathology related to sporadicParkinson's disease. Neurobiol Aging 2003, 24:19721.

Brundin P, Melki R, Kopito R. Prion-like transmission of protein aggregates in neurodegenerative diseases. Nat Rev Mol Cell Biol. 2010 Apr;11(4):301-7.

Cioni JM, Lin JQ, Holtermann AV, Koppers M, Jakobs MAH, Azizi A, Turner-Bridger B, Shigeoka T, Franze K, Harris WA, Holt CE. Late Endosomes Act as mRNA Translation Platforms and Sustain Mitochondria in Axons. Cell. 2019 Jan 10;176(1-2):56-72.e15.

Encalada SE, Goldstein LS. Biophysical challenges to axonal transport: motor-cargo deficiencies and neurodegeneration. Annu Rev Biophys. 2014;43:141-69

Fernandopulle MS, Lippincott-Schwartz J, Ward ME. RNA transport and local translation in neurodevelopmental and neurodegenerative disease. Nat Neurosci. 2021 Jan 28. 
Ghee M, Melki R, Michot N, Mallet J. PA700, the regulatory complex of the 26S proteasome, interferes with alpha-synuclein assembly. FEBS J. 2005; 272:4023-4033.

Golde, T. E., Borchelt, D. R., Giasson, B. I. \& Lewis, J. Thinking laterally about neurodegenerative proteinopathies. J. Clin. Invest. 2013; 123:1847-1855.

Gowrishankar S, Yuan P, Wu Y, Schrag M, Paradise S, Grutzendler J, De Camilli P, Ferguson SM. Massive accumulation of luminal protease-deficient axonal lysosomes at Alzheimer's disease amyloid plaques. Proc Natl Acad Sci U S A. 2015 Jul 14;112(28):E3699-708.

Guo W, Stoklund Dittlau K, Van Den Bosch L. Axonal transport defects and neurodegeneration: Molecular mechanisms and therapeutic implications. Semin Cell Dev Biol. 2020 Mar;99:133-150.

Hardy J, Revesz T. The spread of neurodegenerative disease. N Engl J Med. 2012 May $31 ; 366(22): 2126-8$.

Haziza S, Mohan N, Loe-Mie Y, Lepagnol-Bestel AM, Massou S, Adam MP, Le XL, Viard J, Plancon C, Daudin R, Koebel P, Dorard E, Rose C, Hsieh FJ, Wu CC, Potier B, Herault Y, Sala C, Corvin A, Allinquant B, Chang HC, Treussart F, Simonneau M. Fluorescent nanodiamond tracking reveals intraneuronal transport abnormalities induced by brain-disease-related genetic risk factors. Nat Nanotechnol. 2017 May;12(4):322-328.

Jucker M, Walker LC. Propagation and spread of pathogenic protein assemblies in neurodegenerative diseases. Nat Neurosci. 2018 Oct;21(10):1341-1349. doi: 10.1038/s41593-018-0238-6.

Lee WC, Yoshihara M, Littleton JT. Cytoplasmic aggregates trap polyglutaminecontaining proteins and block axonal transport in a Drosophila model of Huntington's disease. Proc Natl Acad Sci U S A. 2004 Mar 2;101(9):3224-9.

Li X, Tsolis KC, Koper MJ, Ronisz A, Ospitalieri S, von Arnim CAF, Vandenberghe R, Tousseyn T, Scheuerle A, Economou A, Carpentier S, Otto M, Thal DR. Sequence of proteome profiles in preclinical and symptomatic Alzheimer's disease. Alzheimers Dement. 2021 Apr 19. 
Liao YC, Fernandopulle MS, Wang G, Choi H, Hao L, Drerup CM, Patel R, Qamar S, Nixon-Abell J, Shen Y, Meadows W, Vendruscolo M, Knowles TPJ, Nelson M, Czekalska MA, Musteikyte G, Gachechiladze MA, Stephens CA, Pasolli HA, Forrest LR, St George-Hyslop P, Lippincott-Schwartz J, Ward ME. RNA Granules Hitchhike on Lysosomes for Long-Distance Transport, Using Annexin A11 as a Molecular Tether. Cell. 2019 Sep 19;179(1):147-164.e20.

Marshall KE, Vadukul DM, Staras K, Serpell LC. Misfolded amyloid-beta-42 impairs the endosomal-lysosomal pathway. Cell Mol Life Sci. 2020 Dec;77(23):5031-5043.

De Matteis MA, Luini A. Mendelian disorders of membrane trafficking. N Engl J Med. 2011 Sep 8;365(10):927-38.

Millecamps S, Julien JP. Axonal transport deficits and neurodegenerative diseases. Nat Rev Neurosci. 2013 Mar;14(3):161-76.

Morfini GA, Matthew Burns, Lester I Binder, Nicholas M Kanaan, Nichole LaPointe, Daryl A Bosco, Robert H Brown Jr, Hannah Brown, Ashutosh Tiwari, Lawrence Hayward, Julia Edgar, Klaus-Armin Nave, James Garberrn, Yuka Atagi, Yuyu Song, Gustavo Pigino, Scott T Brady. Axonal transport defects in neurodegenerative diseases. J Neurosci 2009 Oct 14;29(41):12776-86.

Peelaerts W, Bousset L, Van der Perren A, Moskalyuk A, Pulizzi R, Giugliano M, Van den Haute C, Melki R, Baekelandt V. a-Synuclein strains cause distinct synucleinopathies after local and systemic administration. Nature. 2015 Jun $18 ; 522(7556): 340-4$.

Renner, M., Lacor, P.N., Velasco, P.T., Xu, J., Contractor, A., Klein, W.L., and Triller, A. (2010). Deleterious effects of amyloid b oligomers acting as an extracellular scaffold for mGluR5. Neuron 66, 739-754.Saez-Atienzar S, Masliah E. Cellular senescence and Alzheimer disease: the egg and the chicken scenario. Nat Rev Neurosci. 2020 Aug;21(8):433-444.

Saudou, F, Humbert, S. (2016) The Biology of Huntingtin. Neuron 89, Issue 5, 2 March 2016, Pages 910-926.

Shrivastava AN, Aperia A, Melki R, Triller A. Physico-Pathologic Mechanisms Involved in Neurodegeneration: Misfolded Protein-Plasma Membrane Interactions. Neuron. 2017 Jul 5;95(1):33-50. 
Shrivastava AN, Bousset L, Renner M, Redeker V, Savistchenko J, Triller A, Melki R. Differential Membrane Binding and Seeding of Distinct $\alpha$-Synuclein Fibrillar Polymorphs. Biophys J. 2020 Mar 24;118(6):1301-1320.

Shrivastava, A.N., Kowalewski, J.M., Renner, M., Bousset, L., Koulakoff, A.,Melki, R., Giaume, C., and Triller, A. (2013). b-amyloid and ATP-induced diffusional trapping of astrocyte and neuronal metabotropic glutamate type-5 receptors. Glia 61, 1673-1686.

Shrivastava AN, Redeker V, Fritz N, Pieri L, Almeida LG, Spolidoro M, Liebmann T, Bousset L, Renner M, Léna C, Aperia A, Melki R, Triller A. alpha-synuclein assemblies sequester neuronal alpha3-Na+/K+-ATPase and impair $\mathrm{Na}+$ gradient. EMBO J. 2015 Oct 1;34(19):2408-23.

Shrivastava AN, Redeker V, Pieri L, Bousset L, Renner M, Madiona K, Mailhes-Hamon C, Coens A, Buée L, Hantraye P, Triller A, Melki R. Clustering of Tau fibrils impairs the synaptic composition of $\alpha 3-\mathrm{Na}+/ \mathrm{K}_{+}-\mathrm{ATP}$ ase and AMPA receptors. EMBO J. 2019 Feb $1 ; 38(3): e 99871$.

Soto, C. \& Pritzkow, S. Protein misfolding, aggregation, and conformational strains in neurodegenerative diseases. Nat. Neurosci. 2018; 21:1332-1340.

Stokin G.B., Lillo C., Falzone T.L., Brusch R.G., Rockenstein E., Mount S.L., Raman R., Davies P., Masliah E., Williams D.S., Goldstein, L.S.. Axonopathy and transport deficits early in the pathogenesis of Alzheimer's disease. Science. 2005;307:12821288.

Valm AM, Cohen S, Legant WR, Melunis J, Hershberg U, Wait E, Cohen AR, Davidson MW, Betzig E, Lippincott-Schwartz J. Applying systems-level spectral imaging and analysis to reveal the organelle interactome. Nature. 2017 Jun 1;546(7656):162-167.

Victoria GS, Zurzolo C. The spread of prion-like proteins by lysosomes and tunneling nanotubes: Implications for neurodegenerative diseases. J Cell Biol. 2017 Sep $4 ; 216(9): 2633-2644$.

Volpicelli-Daley LA, Gamble KL, Schultheiss CE, Riddle DM, West AB, Lee VM. Parkinson's disease Formation of $\alpha$-synuclein Lewy neurite-like aggregates in axons impedes the transport of distinct endosomes. Mol Biol Cell. 2014 Dec $15 ; 25(25): 4010-23$. 
Walker LC, Jucker M. Neurodegenerative diseases: expanding the prion concept. Annu Rev Neurosci. 2015 Jul 8;38:87-103.

Walsh DM, Thulin E, Minogue AM, Gustavsson N, Pang E, Teplow DB, Linse S (2009) A facile method for expression and purification of the Alzheimer's disease-associated amyloid beta-peptide. FEBS J 276:1266-1281. 


\section{Material \& methods}

\section{Production of $\alpha$-syn fibrillar assemblies, Aß fibrils and oligomers}

The expression and purification of human WT $\alpha$-syn was performed as previously described (Ghee et al., 2005). Pure WT $\alpha$-syn was incubated in buffer A to obtain the fibrillar polymorph "fibrils" $\alpha$-synF (50 mM Tris- $\mathrm{HCl}$ at $\mathrm{pH} 7.5,150 \mathrm{mM} \mathrm{KCl})$ and in buffer B for "ribbons" $\alpha$-synR (5 $\mathrm{mM}$ Tris- $\mathrm{HCl}$ at $\mathrm{pH} 7.5$ ) at $37^{\circ} \mathrm{C}$ under continuous shaking in an Eppendorf Thermomixer (Hamburg, Germany) set at 600 rotations per minute (rpm) for 4-7 days (Bousset et al., 2013). The fibrillar $\alpha$-syn polymorphs were centrifuged twice at $15,000 \mathrm{~g}$ for $10 \mathrm{~min}$ and resuspended twice in phosphate-buffered saline (PBS) at 1,446 $\mathrm{g} / \mathrm{L}$ prior to labeling with ATTO $488 \mathrm{NHS}$ ester (\#AD 488-3, Atto-Tec, Siegen, Germany) fluorophore following the manufacturer's instructions using a protein/dye ratio of $1: 2$. The labeling reactions were arrested by addition of $1 \mathrm{mM}$ Tris $(\mathrm{pH}$ 7.5). The unreacted fluorophore was removed by a final cycle of two centrifugations at $15,000 \mathrm{~g}$ for $10 \mathrm{~min}$ and resuspensions of the pellets in PBS. This labeling protocol typically yields $\geq 1$ ATTO molecule incorporated per $\alpha$-syn monomer on average as previously demonstrated (Shrivastava et al., 2015). The assemblies were examined by transmission electron microscopy after adsorption on 200 mesh carbon-coated electron microscopy grids and negative stained with 1\% uranyl acetate before and after fragmentation using a JEOL 1400 electron microscope (JEOL, Tokyo, Japan).

The expression and purification of Met-Aß 1-42 was performed as described (Walsh et al., 2009). $A B$ was assembled in $P B S$, at $4^{\circ} \mathrm{C}$ or $37^{\circ} \mathrm{C}$ without shaking for 2 or $24 \mathrm{~h}$ to obtain oligomers $A B O$ or fibrils $A B F$, respectively. The two kinds of assemblies were labeled with ATTO $488 \mathrm{NHS}$-ester at a protein/dye ratio of 1:2. The labeling reactions were arrested by addition of $1 \mathrm{mM}$ Tris at $\mathrm{pH}$ 7.5. For fibrillar $A ß$, the unreacted fluorophore was removed by two cycles of centrifugation and resuspension of the pelleted fibrils in PBS as described for $\alpha-$ syn. For oligomeric $A ß$, the oligomers were separated from the monomeric and fibrillar forms of the protein by size exclusion chromatography on a Superose $6 \mathrm{HR} 10 / 300$ column (GE Healthcare, Life Sciences, Wauwatosa, WI, USA) equilibrated in PBS pH 7.4 at a flow rate of $0.5 \mathrm{~mL} / \mathrm{min}$. Elution was monitored by measuring absorbance at $280 \mathrm{~nm}$ wavelength. The Superose 6 column was calibrated with Dextran blue (over $2200 \mathrm{kDa}),(670 \mathrm{kDa}), \beta$-amylase (200 kDa), BSA (66 kDa), and carbonic anhydrase (29 kDa) standards (Sigma-Aldrich).

\section{Primary mouse cortical neuron cultures}

We used commercial primary mouse cortical neurons (ref. Gibco A15586, ThermoFisher) because the provider quality check guarantees a purity of $98 \%$ of neurons. The cells were grown on high optical quality glass coverslips (high-precision $170 \pm 5 \mu \mathrm{m}$ thick, $18 \mathrm{~mm}$ diameter, ref. 0117580 , Marienfeld $\mathrm{GmbH}$, Germany). The coverslips are first cleaned with $70 \%$ ethanol, rinsed with water for injection (ref. A128730, ThermoFisher Inc., USA) and exposed during $1 \mathrm{~h}$ to UV light. They were then coated with $0.1 \mathrm{mg} / \mathrm{ml}$ poly-L-ornithine (ref. P3655, Sigma-Aldrich Merck KGaA, Germany) and placed for $2 \mathrm{~h}$ in an incubator set at $37^{\circ} \mathrm{C}$, then rinsed twice with water and let dry at biological hood for one hour. We plated an amount of $6 \times 10^{5}$ primary mouse cortical neurons (ref. Gibco A15586, ThermoFisher) on each coated coverslip, which was then put at the bottom of a 6-wells plate, each well-being finally filled with $3 \mathrm{~mL}$ of neurobasal phenol red-free medium (ref. 12348017, ThermoFisher) containing $0.5 \mathrm{mM}$ GlutaMax (ref. 35050061 ThermoFisher), 2\% B-27 (ref. 17504044, ThermoFisher) and 1\% PenStrep (ref. 15070063, ThermoFisher). The 6-well plate was then placed in an incubator at $37{ }^{\circ} \mathrm{C}$ and $5 \% \mathrm{CO}_{2}$. Half of the volume of the medium was replaced with fresh medium $24 \mathrm{~h}$ after plating. We made the 
subsequent medium changes every 3 days to reduce glutamate toxicity. Neurons were grown until 21 days in culture.

\section{Exposure of mouse cortical neurons to $\alpha$-syn fibrillar assemblies, $A \beta$ fibrils or oligomers}

In all the measurements dealing with (1) the impact of pathogenic protein assemblies on endosomal-lysosomal transport, (2) their colocalization with FND-labelled compartment or lysosomes, or (3) the tracking of their intraneuronal transport by fluorescence videomicroscopy, cortical neurons were incubated with either $0.2 \mu \mathrm{M}$ ATTO 488-labeled $\alpha$ synF or R, or $1 \mu \mathrm{M}$ ATTO 488-labeled ABF or ABO.

$\alpha$-synF or $R$ were added at $24 \mathrm{~h}, 48 \mathrm{~h}$, or $72 \mathrm{~h}$ before observations, while the addition time was either $24 \mathrm{~h}$ or $48 \mathrm{~h}$ for ATTO 488 -labeled $\mathrm{ABF}$ or $\mathrm{ABO}$. The video acquisitions of all the experiments were performed at DIC21.

\section{Intraneuronal transport cargo labeling}

To evaluate the endosomal transport parameters, we relied on our fluorescent nanodiamond assay (Haziza et al. 2017). We used commercially available sized $35 \mathrm{~nm}$ FND (brFND-35, FND Biotech, Taiwan). Each NP contains an average of 15 nitrogen-vacancy emitters displaying a peak emission wavelength around $700 \mathrm{~nm}$ and a full-width at half-maximum of $\approx 100 \mathrm{~nm}$. This far-red emission allows also to investigate the colocalization of green-emitting ATTO 488labelled neurodegenerative-disease related species with FND-labeled cargos. FND were internalized in cortical neurons just before the transport analysis, at DIC21. Each culture coverslip was removed from the 6-well plate containing maintaining medium and put in contact with $400 \mu \mathrm{L}$ of fresh culture medium to which we added $2 \mu \mathrm{l}$ of stock solution of FNDs $(1 \mathrm{mg} / \mathrm{mL}$ ), reaching a final FND concentration of $5 \mu \mathrm{g} / \mathrm{mL}$. After 10 mins incubation, the extra FND-containing medium was absorbed by a wiper sheet and the coverslip was placed back to the dish containing the old maintaining medium. The culture was then placed back during 20 mins in the incubator before the video acquisition.

To measure lysosomal transport or investigate the colocalization of neurodegenerative disease-related species with lysosomes, cortical neurons were stained at DIC21, just before the observation, with LysoTracker Deep Red (ref. L12492, ThermoFisher). This dye molecule has an emission spectrum within the similar range than the one of FND. The coverslip was removed from maintaining medium and incubated with prewarmed $\left(37^{\circ} \mathrm{C}\right)$ culture medium containing $50 \mathrm{nM}$ LysoTracker for $1 \mathrm{~h}$. The probe-containing medium was replaced with the old maintaining medium and followed by video acquisition.

\section{Pseudo-total internal reflection (TIRF) live-cell videomicroscopy}

Pseudo-TIRF illumination was implemented on an inverted microscope (Eclipse Ti-E, Nikon, Japan) as described in details in (Haziza et al. 2017). The whole microscope is enclosed in a cage incubator (Okolab, Italy) to maintain temperature at $37^{\circ} \mathrm{C}$. For the intraneuronal transport recording, each coverslip supporting the neuron culture is mounted at the bottom a Ludin chamber (type 1, Life Imaging Service, Switzerland), installed inside the environmental chamber (in which $5 \%$ partial $\mathrm{CO}_{2}$ pressure and $100 \%$ hydrometry is maintained) having a hole at its bottom allowing direct optical access of the microscope objective to the coverslip. We used $\mathrm{a} \times 100$ magnification and 1.49 numerical aperture immersion oil objective (CFI Apo TIRF $\times 100$ Oil, Nikon), compatible with differential inference contrast (DIC) mode. Field of views of interest of the neuron cultures were selected in white-light illumination DIC mode. Two continuous-wave lasers are coupled to the microscope and fluorescence was recorded on a cooled EMCCD array detector (DU-885K-CS0, Andor Technologies, UK) of $1004 \times 1002$ pixels, 
with $80 \mathrm{~nm}$ pixel size in the sample plane. Two-minutes duration videos were acquired at 20 full frame/s rate large enough to be able to detect short pausing duration in cargoes displacements. EMCCD parameters were selected to provide the largest signal-to-background ratio for FND label tracking at the selected frame rate, leading to EM gain of 90, preamplification gain $\times 3.8$, and digitalization speed of $35 \mathrm{MHz}$. FNDs and Lysotracker fluorophore were excited with a diode-pumped solid-state laser at a wavelength of $561 \mathrm{~nm}$ (SLIM-561-100, Oxxius S.A., France), while ATTO 488 dye was excited with a laser diode emitting at a wavelength of $488 \mathrm{~nm}$ (LBX-488-200-CSB-PP, Oxxius S.A). Each excitation laser power was adjusted so that the detection dynamic range of all channels was identical for the above mentioned fixed EMCCD settings. This leads to $561 \mathrm{~nm}$ laser excitation power of $60 \mathrm{~mW}$ or $200 \mu \mathrm{W}$ for FND or LysoTracker respectively, and to $488 \mathrm{~nm}$ laser excitation power of $200 \mu \mathrm{W}$ for ATTO 488 dye.

To perform two-color acquisitions and record simultaneously FND (or LysoTracker) and ATTO 488 we combined the two laser beams with a dual-band dichroic filter (ref. Di01-R488/561, Semrock, USA), and placed a dual imaging system (W-VIEW GEMINI, Hamamatsu, Japan) in front of the EMCCD array detector (DU-885K-CS0, Andor Technologies, UK). This system splits half of the detection field of view (FoV) in two color channels with a dichroic beamsplitter (FF560-FDi01, Semrock) and projects each color on half of the array detector, further preceded by bandpass detection filters (red channel: HC697/75, Semrock; green channel: ET525/50, Chroma Corporation, USA). The result is that each frame of the video contains the same rectangular FoV $(1004 \times 501$ pixels) in green (ATTO 488) and red (FND and LysoTracker) emission range, allowing to identify spots that colocalize dynamically.

\section{Video processing and intraneuronal transport quantification}

Two programs written in python were developed to extract quantitative parameters from videos automatically. The first one relies on Trackpy 0.4.2 package (Trackpy 2019), from which it uses two functions: locate to identify isolated spots in each fluorescence frame and fit them with gaussians, and link which connects the spots between frames to form trajectories using Crocker-Grier algorithm (Crocker \& Grier 1996). Transport parameters are then calculated with a second program which first parses each trajectory into "go" and "stop" phases based on the confinement ratio calculation as described in (Haziza et al., 2017). Four main transport parameters are extracted for each trajectory: velocity, which is the average speed of all go phases; run length: average distance traveled during all go phases; pausing time: average duration of the stop phases, and pausing frequency (events/min). In addition to these four main parameters, we also calculated the total length of the trajectory as the sum of all run lengths during go phases.

\section{Lysosomes size estimation}

To get an estimate of the lysosome size from the diffraction limited fluorescence images, we considered the LysoTracker spots as the result of the convolution of the microscope point spread function (assimilated to a gaussian of standard deviation, SD, $\sigma_{\mathrm{PSF}}$ ) and the lysosome assimilated to a symmetrical gaussian of SD $\sigma_{\mathrm{L}}$. The result of this convolution is also a gaussian of SD $\sigma_{\mathrm{T}}$, related to $\sigma_{\mathrm{L}}$ and $\sigma_{\mathrm{PSF}}$ by ${\sigma_{\mathrm{T}}}^{2}={\sigma_{\mathrm{PSF}}}^{2}+{\sigma_{\mathrm{L}}^{2}}^{2} \cdot \sigma_{\mathrm{T}}$ is an output of the Trackpy locate function. The knowledge of $\sigma_{\mathrm{T}}$ and $\sigma_{\mathrm{PSF}}$ allows the derivation of $\sigma_{\mathrm{L}}$. We then defined the lysosome "diameter" $d_{\mathrm{L}}$ as the full width at half maximum of its gaussian approximation, inferred by $d_{\mathrm{L}}=2 \sqrt{2 \ln 2} \sigma_{\mathrm{L}}$. For the PSF size $\sigma_{\mathrm{PSF}}$ we took the measured value of the smallest FND spot observed in several trajectories with our microscope. This value was $\sigma_{\mathrm{PSF}}=112 \mathrm{~nm}$, consistent with the theoretical Airy radius $\rho_{A}=286 \mathrm{~nm}$ (diffraction limit at $700 \mathrm{~nm}$ maximum 
emission wavelength for the 1.49 numerical aperture objective used), and the empirical relation $\sigma_{\mathrm{PSF}}=\frac{\rho_{\mathrm{A}}}{3}$, giving here an experimental PSF SD of $286 / 3=95 \mathrm{~nm}$.

\section{Data representation and statistical analysis}

All bar plots display the \pm standard error on the mean of the distribution. Box plots display the median value as the horizontal line within the box whose limits are $25 \%$ and $75 \%$ percentiles; bottom and top horizontal lines correspond to $10 \%$ and $90 \%$ percentiles. As all the data compared between two conditions were random and normally distributed but with unequal variance (as tested with a $F$-test), we performed the relevant comparison test which is the nonparametric Wilcoxon Mann-Whitney two-tailed (implemented in Igor Pro 8, Wavemetrics Inc., USA). Stars referred to the following $p$-value significance level: ${ }^{*} p<0.05 ;{ }^{* *} p<0.01 ;{ }^{* * *} p<0.001$.

\section{References}

(Crocker \& Grier 1996) Crocker JC, Grier DG (1996) Methods of Digital Video Microscopy for Colloidal Studies. J Colloid Interf Sci 179:298-310.

(Trackpy 2019) doi: 10.5281/zenodo.3492186 


\section{Supplementary Information}

\section{Supplementary Figures}
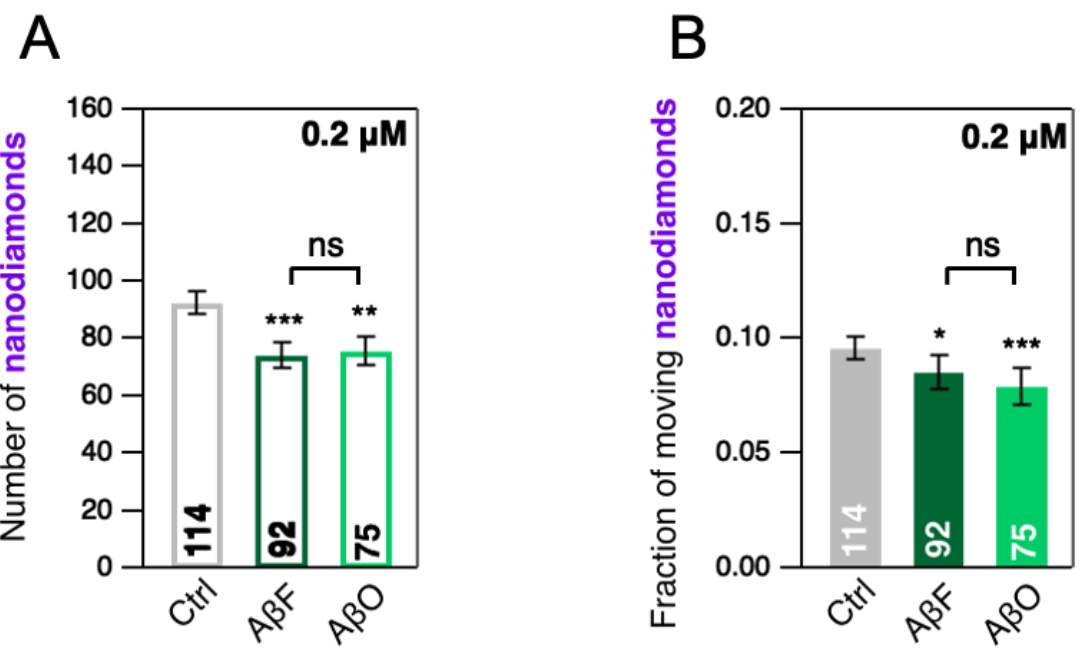

Figure S1. Impact of $A \beta$ fibrils and oligomers at the concentration of $0.2 \mu \mathrm{M}$ on the number of nanodiamonds interacting with cells $(\mathrm{A})$ and on the fraction of moving nanodiamonds $(\mathrm{B})$.

A
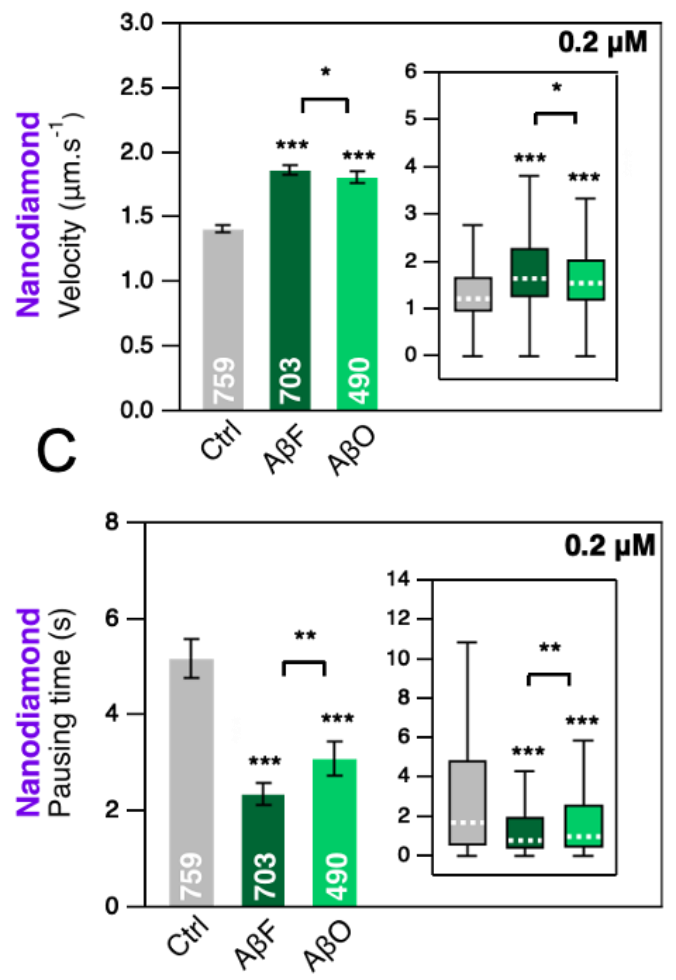

B
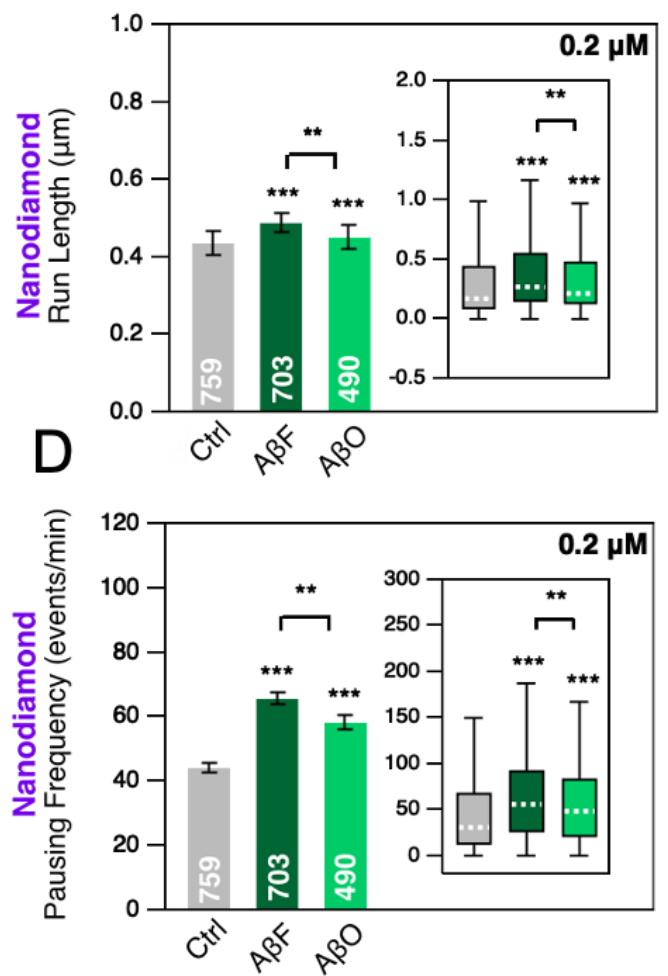

Figure S2. Impact of $A \beta F$ and $A B O$ at the concentration of $0.2 \mu \mathrm{M}$ on FND-labelled endosome transport parameters. 


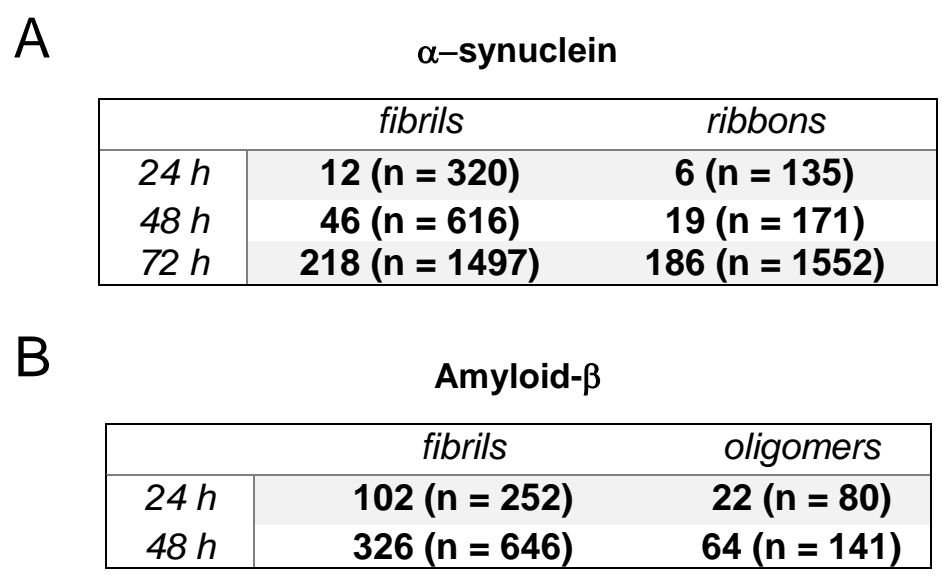

Figure S3. Number of neurodegenerative-related molecular species trajectories colocalized with Iysosomes as a function of time. A) $\alpha$-synuclein. B) amyloid- $\beta . n$ represents the total number of trajectories. The numbers of trajectories in each time points were analyzed from 2 coverslips (from one culture). 
A $\quad 0.2 \mu \mathrm{M} \alpha$-syn Fibrils
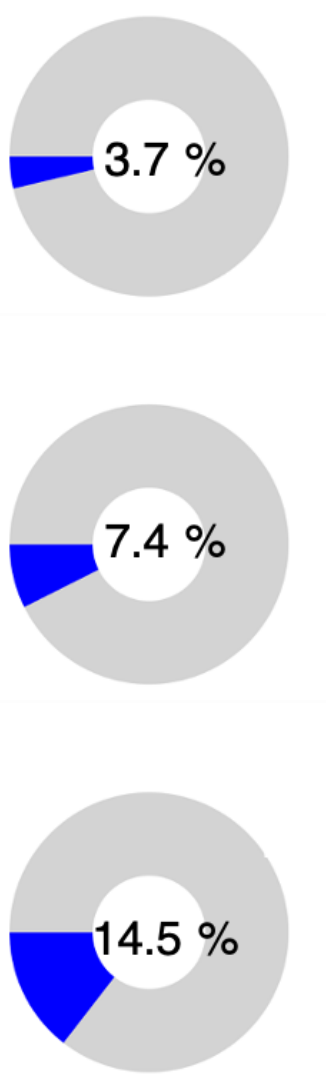

B

\section{$0.2 \mu \mathrm{M} \alpha$-syn Ribbons}

$24 \mathrm{~h}$

\section{$4.4 \%$}

$48 h$

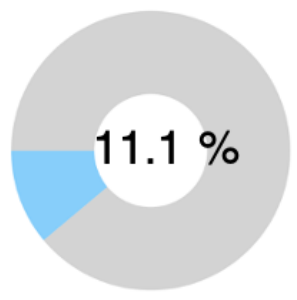

$72 \mathrm{~h}$

$11.9 \%$
$1 \mu \mathrm{M}$ A $\beta$ Fibrils
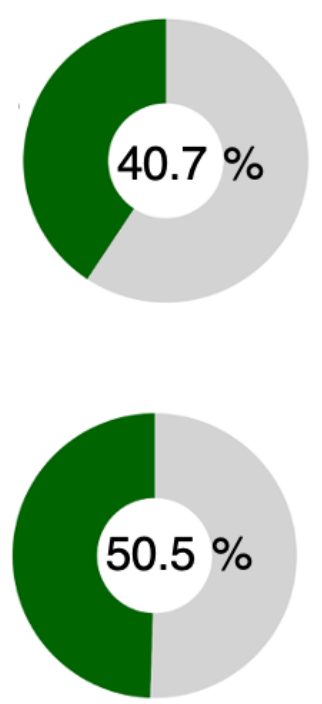

$24 \mathrm{~h}$

$1 \mu \mathrm{M}$ A $\beta$ Oligomers

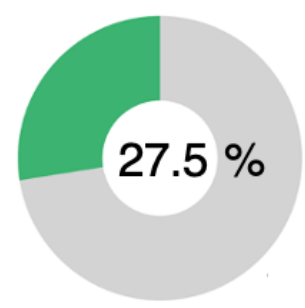

$48 \mathrm{~h}$

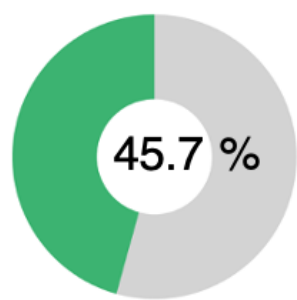

Figure S4. Colocalized events between moving $\alpha$-synF, $\alpha$-synR, ABF, $A B O$ and moving lysosomes at different time points. A) $\alpha$-synF and $\alpha$-synR were incubated at for 24,48 , and $72 \mathrm{~h}$, at concentration of $0.2 \mu \mathrm{M}$. B) ABF and ABO were incubated for 24 , and $48 \mathrm{~h}$, at concentration of $1 \mu \mathrm{M}$. The number inside the donut plot represents the percentage of moving $\alpha$-syn or $A B$ assemblies colocalized with the lysosomes analyzed from $n=2$ coverslips (from one culture). 
bioRxiv preprint doi: https://doi.org/10.1101/2021.05.15.444288; this version posted May 17, 2021. The copyright holder for this preprint (which was not certified by peer review) is the author/funder. All rights reserved. No reuse allowed without permission. 